\title{
HOMOTOPICAL DYNAMICS II: HOPF INVARIANTS, SMOOTHINGS AND THE MORSE COMPLEX
}

\author{
BY OCTAVIAN CORNEA
}

ABSTRACT. - The ambient framed bordism class of the connecting manifold of two consecutive critical points of a Morse-Smale function is estimated by means of a certain Hopf invariant. Applications include new examples of non-smoothable Poincaré duality spaces as well as an extension of the Morse complex.

(c) 2002 Éditions scientifiques et médicales Elsevier SAS

RÉSUMÉ. - La classe ambiante de bordisme stablement parallélisé de la variété des trajectoires hétéroclines associée à deux points critiques successifs d'une fonction de Morse-Smale est calculée en fonction d'un certain invariant de Hopf. Dans les applications dont nous traitons, on trouvera notamment la construction de nouveaux exemples d'espaces à dualité de Poincaré qui ne sont pas lissables et une généralisation du complexe de Morse.

(C) 2002 Éditions scientifiques et médicales Elsevier SAS

\section{Introduction}

Let $M$ be a smooth, connected, compact, riemannian manifold and let $f: M \longrightarrow \mathbf{R}$ be a smooth Morse-Smale function, regular and constant on $\partial M$. The flow $\gamma: M \times \mathbf{R} \longrightarrow M$ used below is induced by $-\nabla f$. Assume that $P$ and $Q$ are consecutive critical points of $f$ (this means that $f(P)>f(Q)$ and that there are no broken flow lines connecting $P$ to $Q$ ) of indices, respectively, $p$ and $q$.

An important, classical problem in Morse theory is to use the topology of $M$ to understand the properties of the moduli space $Z(P, Q)$ of flow lines that connect $P$ to $Q$.

This problem is the main motivation of the paper. The key new idea introduced here is that knowledge of the homotopy of the based loop space $\Omega M$ leads to significant information on these moduli spaces.

More precisely, recall that the genericity of the Morse-Smale condition implies that $Z(P, Q)$ is a manifold of dimension $p-q-1$ called connecting manifold of $P$ and $Q$. It has a canonical normal framing and a classical result of John Franks [11] claims that its framed bordism class $\{Z(P, Q)\} \in \Omega_{*}^{f r}$ is given (via the Thom-Pontryagin construction) by the relative attaching map $\delta_{f}(P, Q)$ associated to the succesive attachments of the cells corresponding to the critical points $Q$ and $P$.

The closure of the space of all the points situated on some flow line joining $P$ to $Q$ is identified to the unreduced suspension $\Sigma Z(P, Q)$. Therefore, we have an inclusion $\Sigma Z(P, Q) \hookrightarrow M$ and, after fixing a base-point in $Z(P, Q)$ and in $M$, we get an adjoint

$$
l(P, Q): Z(P, Q) \longrightarrow \Omega M .
$$


The above mentioned normal framing of $Z$ together with the map $l(P, Q)$ provide, via the Thom-Pontryagin construction, a homotopy class

$$
T(P, Q): S^{p-1} \longrightarrow \Sigma^{q}\left(\Omega M^{+}\right)=S^{q} \vee S^{q} \wedge \Omega M
$$

By Franks' result the projection of $T(P, Q)$ on $S^{q}$ is $\delta_{f}(P, Q)$. Let $h(P, Q) \in \pi_{p-1}\left(\Sigma^{q} \Omega M\right)$ be the projection of $T(P, Q)$ on the second factor.

The main result of the paper continues the work of Franks by giving a purely homotopical, computable description of the ambient, framed, bordism class of $Z(P, Q)$,

$$
[Z(P, Q)]^{f r} \in \Omega_{p-q-1}^{f r}(\Omega M)
$$

In fact, we show that $\Sigma h(P, Q)$ equals the suspension of a certain Hopf invariant, $H(P, Q)$, associated to the succesive cell attachments corresponding to $Q$ and $P$. As $T(P, Q)$ represents $[Z(P, Q)]^{f r}$ we conclude that this bordism class equals the stable image of $H(P, Q)+\delta_{f}(P, Q)$.

The homotopy classes $h(P, Q)$ turn out to be highly relevant for understanding the respective connecting manifolds and also for the topology of $M$ itself. We study some of their properties.

Specializing to the Morse-Smale case the Spanier-Whitehead duality results of [6,7], we show that, stably, $\varepsilon \delta_{-f}(Q, P)$ equals $\delta_{f}(P, Q)+\Delta(P, Q)$ where $\varepsilon \in\{-1,+1\}$ with the twisting term

$$
\Delta(P, Q)=J^{q}\left(\Omega^{q} \Sigma^{q}(\Omega \nu) \circ h^{*}(P, Q)\right) .
$$

Here $\nu: M \longrightarrow$ BSO classifies the stable normal bundle of $M, h^{*}(P, Q)$ is the $q$ th order adjoint of $h(P, Q)$ and $J^{q}: \pi_{k}\left(\Omega^{q} \Sigma^{q} \mathbf{S O}\right) \longrightarrow \pi_{k}^{S}$ is a factor of the classical J-homomorphism $J: \pi_{k}(\mathbf{S O}) \longrightarrow \pi_{k}^{S}$. This can be used to deduce a measure of the embedding complexity of $Z(P, Q)$. We also use this result to construct examples of non-smoothable Poincaré duality spaces (many of which are PL-manifolds). When the obstructions to smoothing concern only the relative attaching maps of the top cell the relevant morphism is, of course, $J=J^{0}$ and one recovers in this case classical results (see for instance [35]) and the examples of Smith [34] which are thus seen to be part of a more general pattern.

In a different direction, if $P, R$ are critical points such that $f(P)>f(R)$ and all flow lines connecting $P$ to $R$ are broken at most once, let $I(P, R)$ be the set of intermediate critical points. We show that, when $M$ is simply-connected, the relation

$$
\sum_{Q \in I(P, R)}(-1)^{(p+r) q}[Z(P, Q)]^{f r} \bullet[Z(Q, R)]^{f r}=0
$$

is satisfied inside the ring $\Omega_{*}^{f r}(\Omega M)$ (where the product $\bullet$ is induced by loop composition). This can be used to define various chain complexes which, in the simplest possible case (when the index of successive critical points differs by just one), provide the Morse complex of $f$.

The material is organized as follows. After a second section, containing a review of known facts and fixing notation, comes the technical heart of the paper, in section three, very much in the spirit of the work of Franks [11]. The description of $\Sigma h(P, Q)$ in terms of the Hopf invariant is given at this point, as well as the proof of the relations among the bordism classes of the connecting manifolds. The fourth section contains applications and most homotopy theoretical arguments are concentrated here. 


\section{Review and notation}

\subsection{Hopf invariants}

For further use we fix some standard facts. We denote by $A * B$ the join of $A$ and $B$. For pointed spaces, $A * B \simeq \Sigma A \wedge B, \Sigma A=S^{1} \wedge A$. Recall also that $A^{+}$is the pointed space obtained by the disjoint union of $A$ and a disjoint base point; if $A$ is pointed we have $(A \times B) /(* \times B)=A \wedge B^{+}$. For $A$ and $B$ connected we will also need the existence of the homotopy fibration $\Omega A * \Omega B \longrightarrow A \vee B \longrightarrow A \times B$ which is trivial after looping.

Assume that $X$ is a pointed, connected $C W$-complex and that $X^{\prime} \hookrightarrow X^{\prime \prime} \stackrel{i}{\hookrightarrow} X$ are pointed inclusions of connected subcomplexes such that there is a cofibration sequence

$$
S^{q-1} \stackrel{f}{\longrightarrow} X^{\prime} \longrightarrow X^{\prime \prime}
$$

of pointed spaces. Fix also a pointed map $S^{p-1} \stackrel{g}{\longrightarrow} X^{\prime \prime}$.

The Hopf invariants that we will use are defined using this data (they are versions of invariants defined by Ganea [12,9]).

The Hopf invariant of $g$ relative to $f$ is obtained as follows. Consider the map

$$
t: S^{p-2} \longrightarrow \Omega S^{p-1} \stackrel{\Omega g}{\longrightarrow} \Omega X^{\prime \prime} \stackrel{\Omega \nabla}{\longrightarrow} \Omega\left(S^{q} \vee X^{\prime \prime}\right) \stackrel{p}{\longrightarrow} \Omega\left(\Omega S^{q} * \Omega X^{\prime \prime}\right) .
$$

Here, $\nabla$ is the coaction $X^{\prime \prime} \longrightarrow S^{q} \vee X^{\prime \prime}$ and $p$ is the canonical projection in the splitting $\Omega\left(S^{q} \vee X^{\prime \prime}\right) \simeq \Omega S^{q} \times \Omega X^{\prime \prime} \times \Omega\left(\Omega S^{q} * \Omega X^{\prime \prime}\right)$; the first map in the composition is the restriction to the bottom cell.

We have a projection $r: \Omega S^{q} * \Omega X^{\prime \prime} \longrightarrow S^{q} \wedge \Omega X^{\prime \prime}$ induced by the canonical evaluation $\Sigma \Omega S^{q} \longrightarrow S^{q}$.

The needed Hopf invariant is the homotopy class:

$$
H(g, f)=\left(i d_{S^{q}} \wedge \Omega i\right) \circ r \circ t^{*}: S^{p-1} \longrightarrow S^{q} \wedge \Omega X
$$

where $t^{*}: S^{p-1} \longrightarrow \Omega S^{q} * \Omega X^{\prime \prime}$ is the adjoint of $t$.

We denote by $\delta=\delta(f, g): S^{p-1} \longrightarrow S^{q}$ the relative attaching map given by projecting $\nabla \circ g$ onto $S^{q}$.

Remark 1. - For example, let $*: S^{1} \longrightarrow *$ and let $\eta: S^{3} \longrightarrow S^{2}$ be the Hopf map and $X=\mathbf{C P}^{2}$. Then $H(\eta, *): S^{3} \longrightarrow S^{2} \wedge \Omega \mathbf{C P}^{2}$ is the inclusion of the bottom cell. Similarly, let $*: S^{q-1} \longrightarrow S^{t}$ be the trivial map and $w: S^{q+t-1} \longrightarrow S^{q} \vee S^{t}$ be the obvious Whitehead product and $X=S^{q} \times S^{t}$. Then $H(w, *): S^{q+t-1} \longrightarrow S^{q} \wedge \Omega\left(S^{q} \times S^{t}\right)$ is the inclusion $S^{q} \wedge S^{t-1} \longrightarrow S^{q} \wedge \Omega S^{t} \longrightarrow S^{q} \wedge\left(\Omega S^{q} \times \Omega S^{t}\right)$.

\subsection{Elements of Morse theory}

We follow here the fundamental paper of John Franks [11].

Let $M^{n}$ be a smooth compact manifold and let $f: M \longrightarrow \mathbf{R}$ be a smooth function. If $\partial M \neq \emptyset$ we assume that the function is constant and regular on $\partial M$. We assume also that a riemannian metric is fixed on $M$ and we denote by $\gamma: M \times \mathbf{R} \longrightarrow M$ the flow induced by $-\nabla f$. A critical point $P$ of $M$ is non-degenerate if $\operatorname{Hess}_{P}(f)$ is a non-degenerate matrix. The index of the 
induced bilinear form is called the index of $P$. The set $W^{u}(P)=\left\{x \in M: \lim _{t \rightarrow-\infty} \gamma_{t}(x)=P\right\}$ is called the unstable manifold of $P$ and $W^{s}(P)=\left\{x \in M: \lim _{t \rightarrow+\infty} \gamma_{t}(x)=P\right\}$ is the stable manifold of $P$. If $P$ is non-degenerate and of index $p$, then $W^{s}(P) \approx \operatorname{Int}\left(D^{n-p}\right)$ and $W^{u}(P) \approx \operatorname{Int}\left(D^{p}\right)$.

We assume from now on that $f$ is Morse which means that all its critical points are nondegenerate, and even Morse-Smale which means that if $P$ and $Q$ are two critical points of $f$, then $W^{u}(P)$ and $W^{s}(Q)$ are in general position. The Morse-Smale condition is generic.

We say that two critical points $P$ and $Q$ are consecutive if $f(P)>f(Q)$ and there are no broken flow lines connecting $P$ to $Q$. In this case, let $f(Q)<a<f(P)$ and let

$$
S^{s}(Q)=W^{s}(Q) \cap f^{-1}(a) \quad \text { and } \quad S^{u}(P)=W^{u}(P) \cap f^{-1}(a) .
$$

If $P$ and $Q$ are critical and consecutive we may (and will) assume also (possibly after slightly isotoping the function $f$ ) that $P$ and $Q$ are the only critical points in $f^{-1}([f(Q), f(P)])$. In this case, it is easy to see that $S^{u}(P) \approx S^{p-1}$ and $S^{s}(Q) \approx S^{n-q-1}$. The Morse-Smale condition insures that $S^{u}(P)$ and $S^{s}(Q)$ intersect transversely. Their intersection, $Z(P, Q)$, is called the connecting manifold of $P$ and $Q$ (a.k.a. the moduli space of flow lines connecting $P$ to $Q$ ). It is a $(p-q-1)$-dimensional manifold. Fix for each critical point $R$ of $f$ an orientation on the linear subspaces $V^{u}(R)$ and $V^{s}(R)$ of $T_{R}(M)$ that are respectively tangent to $W^{u}(R)$ and $W^{s}(R)$. If the manifold $M$ is oriented, pick these orientations such that at each point $R$ they give on $V^{u}(R) \oplus V^{s}(R)$ the fixed orientation of $T_{R}(M)$. It is easy to see that a choice of a basis of $V^{u}(R)$ induces a framing of the normal bundle of $W^{s}(R)$ and, similarly, a choice of basis for $V^{s}(R)$ induces a normal framing of $W^{u}(R)$. We choose these bases in a way compatible with the fixed orientations and then these framings are unique up to isomorphism. Indeed, two normal framings of the same submanifold $G$ of $M$ are isomorphic (and are generally identified as we shall also be doing further in the paper) if they are restrictions to $G \times\{0\}$ and respectively $G \times\{1\}$ of a normal framing of $G \times[0,1]$ in $M \times[0,1]$. In our case, the key fact is that $W^{s}(R)$ (respectively $W^{u}(R)$ ) is contractible and this implies that any two normal framings of $W^{s}(R)$ (respectively $W^{u}(R)$ ) are isomorphic provided they give the same orientation.

We now return to the two consecutive critical points $P$ and $Q$. The normal bundle of $Z(P, Q)$ in $S^{u}(P)$ is induced by the normal bundle of $W^{s}(Q)$ in $M$ and hence, with our choices, it inherits a standard framing (up to isomorphism) [11].

It is well known that the passage through a non-degenerate critical point corresponds to the attachment of a cell of dimension the index of the critical point. In other words, if $M^{\prime}=f^{-1}(-\infty, f(Q)-\varepsilon], M^{\prime \prime}=f^{-1}(-\infty, f(P)-\varepsilon], M^{\prime \prime \prime}=f^{-1}(-\infty, f(P)+\varepsilon]$ with $\varepsilon$ small enough we have homotopy cofibration sequences:

$$
S^{q-1} \stackrel{\alpha(Q)}{\longrightarrow} M^{\prime} \longrightarrow M^{\prime \prime} \quad \text { and } \quad S^{p-1} \stackrel{\alpha(P)}{\longrightarrow} M^{\prime \prime} \longrightarrow M^{\prime \prime \prime}
$$

Denote by $\delta_{f}(P, Q): S^{p-1} \longrightarrow S^{q}$ the obvious relative attaching map.

One of the key results in [11] is that $\delta_{f}(P, Q)$ corresponds to the standard framing of $Z(P, Q)$ in $S^{u}(P)$ via the Thom-Pontryagin construction. When $p-q=1$ this comes down to just counting (with sign) the number of elements in $Z(P, Q)$. Hence, as immediate application of this result of Franks, one obtains that if $C_{i}=\mathbf{Z} / 2<x: \nabla f(x)=0, \operatorname{ind}(x)=i>$ are $\mathbf{Z} / 2$ vector spaces and $d: C_{i} \longrightarrow C_{i-1}$ is the unique linear function defined on basis elements by $d(x)=\sum_{\text {ind }(y)=i-1} \#(Z(x, y)) y$, then $\left(C_{i}, d\right)$ is a complex, called the Morse complex of $f$, and $H_{*}\left(C_{i}, d\right) \simeq H_{*}(M ; \mathbf{Z} / 2)$ (in the oriented case, by using some appropriate signs when counting the elements of $Z(x, y)$ in the definition of $d$, one obtains the integral homology of $M)$. There are at least a couple of other proofs of this fact. An analytical one appears in [41]. Another, that 
will be extended in Sections 3 and 4, is based on understanding the boundaries of certain moduli spaces of connecting flow lines (see for example [31]).

To a smooth Morse-Smale function $f$ on $M$ there corresponds a $C W$-complex with the same homotopy type as $M$ and with one $p$-dimensional cell for each critical point of index $p$ (we consider now only functions that are constant, regular and maximal on $\partial M$ ). If $f$ is smoothly deformed via Morse-Smale functions to a second Morse-Smale function $f^{\prime}$, then the two $C W$-complexes are cell-equivalent in the sense of Franks [11] (which essentially means that they are related by a cellular homotopy equivalence). When the metric is allowed to vary, the cell-equivalence class of the $C W$-complex corresponding to $f$ is determined up to a contractible choice [18].

Conversely, if $M$ and $\partial M$ are simply-connected, $n>5$ and $H_{*}(M ; \mathbf{Z})$ torsion free, then all $C W$-complexes $Y$ of the homotopy type of $M$ and satisfying a certain minimality condition correspond to some Morse-Smale function on $M$ [11] (the minimality condition is the following: $Y$ has a unique 0-dimensional cell, at most one $n$-cell and if $e_{1}^{k}$ and $e_{2}^{l}$ are two cells of $Y$ with $k>l$, then either $2 k-l<n-1$ or the smallest subcomplex of $Y$ that contains $e_{1}^{k}$ contains also $e_{2}^{l}$ ). In particular, there are self-indexed Morse functions having a number of critical points of index $k$ equal to the rank of $H_{k}(M ; \mathbf{Z})$ [33]. They are called perfect Morse functions.

\subsection{Duality and flows}

Let $X$ be a $C W$-complex and consider an orthogonal fiber bundle of rank $n$ over $X$, $\mu: E \longrightarrow X$. Let $E_{S}(X) \longrightarrow X$ be the associated spherical bundle and $T^{\mu}(X)$ the respective Thom space. Assume that for a certain cell-decomposition of $X$ we have cofibration sequences $S^{q-1} \longrightarrow X^{\prime} \longrightarrow X^{\prime \prime}$ and $S^{p-1} \longrightarrow X^{\prime \prime} \longrightarrow X^{\prime \prime \prime}$ with $X^{\prime \prime \prime}$ a subcomplex of $X$. Denote by $\delta: S^{p-1} \longrightarrow S^{q}$ the respective relative attaching map. It is easy to see that the $C W$-decomposition of $X$ induces one for $T^{\mu}(X)$. For example, by pulling back the cofibration sequence $S^{q-1} \longrightarrow X^{\prime} \longrightarrow X^{\prime \prime}$ to the spherical fibration of $\mu$ one obtains a push-out square:

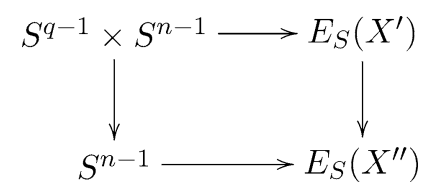

by pushing down this square into the original cofibration we get the push-out square:

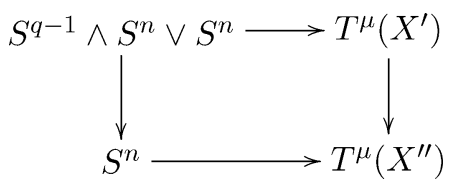

As the left vertical map is just the projection onto $S^{n}$ it is easy to transform this push-out square into a cofibration sequence $S^{n+q-1} \longrightarrow T^{\mu}\left(X^{\prime}\right) \longrightarrow T^{\mu}\left(X^{\prime \prime}\right)$.

In particular we obtain a relative attaching map $\delta^{\mu}: S^{n+p-1} \longrightarrow S^{n+q}$.

We return now to the context and notations of the previous subsection. Thus $f: M \longrightarrow \mathbf{R}$ is a smooth Morse-Smale function; $P$ and $Q$ are consecutive critical points of $f$ of indexes respectively $p$ and $q ; \delta_{f}(P, Q)$ is the corresponding relative attaching map. The function $-f$ is also Morse-Smale and $Q$ and $P$ are consecutive critical points for $-f$. Thus, we also have a relative attaching map $\delta_{-f}(Q, P): S^{n-q-1} \longrightarrow S^{n-p}$.

Let $\nu$ be the stable normal bundle of $M$. The results in [6] imply that $\delta_{f}(P, Q)^{\nu}$ agrees stably up to sign with $\delta_{-f}(Q, P)$ and if $\nu$ is trivial, then $\delta_{f}(P, Q)^{\nu}$ and $\delta_{f}(P, Q)$ agree stably. 
Remark 2. - When $\nu$ is trivial the result appears already in the paper of Franks [11]. In fact, one has much more general results valid for general flows and isolated invariant sets (in the context of Conley index theory) in which the equality up to sign is replaced by SpanierWhitehead duality [6].

\subsection{The J-homomorphism}

We need to recall a few elements of classical homotopy theory. We denote by $\pi_{k}^{S}$ the stable $k$-stem. Similarly, the stable homotopy groups of any space $X$ are denoted by $\pi_{k}^{S}(X)$.

The J-homomorphism $J: \pi_{k}(\mathbf{S O}) \longrightarrow \pi_{k}^{S}$ is defined as follows. For $\alpha \in \pi_{k}(\mathbf{S O})$ there is some $m \in \mathbf{N}$ such that $\alpha \in \pi_{k}(\mathbf{S O}(m))$. Consider the composition

$$
\alpha^{\prime}: S^{m-1} \times S^{k} \stackrel{i d \times \alpha}{\longrightarrow} S^{m-1} \times \mathbf{S O}(m) \stackrel{\cdot}{\longrightarrow} S^{m-1} .
$$

Apply the Hopf construction to this map $\alpha^{\prime}$ (or, equivalently, suspend $\alpha^{\prime}$ and use the splitting of the suspension of the domain to restrict to $S^{m+k}$ ) thus getting a map $\alpha^{\prime \prime}: S^{m+k} \longrightarrow S^{m}$. The image of $\alpha^{\prime \prime}$ in the stable $k$-stem is $J(\alpha)$.

One can show [40] that this construction does not depend of the different choices involved and that the resulting map is a group homomorphism.

As the image of $\mathbf{J}$ is stable, it is immediate to see that this homomorphism factors as $\pi_{k}(\mathbf{S O}) \longrightarrow \pi_{k}^{S}(\mathbf{S O}) \stackrel{J^{\prime}}{\longrightarrow} \pi_{k}^{S}$. The homomorphism $J^{\prime}$ is called the "ery stable $J$ " and also the "bi-stable J-homomorphism" [20]. It is easy to see that, for each $q \geqslant 0$, in between $J$ and $J^{\prime}$ there is an intermediate factor $J^{q}: \pi_{k}\left(\Omega^{q} \Sigma^{q} \mathbf{S O}\right) \longrightarrow \pi_{k}^{S}$. These are also homomorphisms and they commute with the morphisms induced in homotopy by the inclusions

$$
\Omega^{q} \Sigma^{q} \mathbf{S O} \longrightarrow \Omega^{q+1} \Sigma^{q+1} \mathbf{S O}
$$

$J^{0}=J$ and $J^{\prime}$ is the limit of the $J^{q}$ 's.

For later use we give an explicit description of $J^{q}$. Let $\alpha \in \pi_{k}\left(\Omega^{q} \Sigma^{q} \mathbf{S O}\right)$. There is some $m \in \mathbf{N}$ such that $\alpha \in \pi_{k}\left(\Omega^{k} \Sigma^{k}(\mathbf{S O}(m))\right)$. Then $J^{q}(\alpha)$ is the stable image of the composition

$$
S^{k+m+q} \stackrel{\Sigma^{m}\left(\alpha^{*}\right)}{\longrightarrow} \Sigma^{m+q} \mathbf{S O}(m) \stackrel{\mu^{\prime}}{\longrightarrow} S^{m+q}
$$

where $\alpha^{*}$ is the $q$ th order adjoint of $\alpha$ and $\mu^{\prime}$ is the $q$ th suspension of the Hopf construction applied to the multiplication $\mu: S^{m-1} \times \mathbf{S O}(m) \longrightarrow S^{m-1}$.

Remark 3. - The image of J has been computed by Adams [1], Quillen [29] and Sullivan [37]. By the Kahn-Priddy theorem [15] it is known that $J^{\prime}$ is surjective at the prime 2. However, $J^{\prime}$ is not surjective at any odd prime [20].

\section{Morse-theoretic interpretation of the Hopf invariants}

As before, let $M^{n}$ be a smooth, riemannian, compact manifold and $f: M \longrightarrow \mathbf{R}$ a smooth Morse-Smale function regular, maximal and constant on $\partial M$.

We assume from now on that $f$ has a unique local minimum. Suppose that $P$ and $Q$ are consecutive critical points of $f$ (in particular $f(P)>f(Q)$ ) of indexes, respectively, $p$ and $q$.

For $q \geqslant 1$, as in 2.2, consider the induced homotopy cofibration sequences:

$$
S^{q-1} \stackrel{\alpha(Q)}{\longrightarrow} M^{\prime} \longrightarrow M^{\prime \prime} \quad \text { and } \quad S^{p-1} \stackrel{\alpha(P)}{\longrightarrow} M^{\prime \prime} \longrightarrow M^{\prime \prime \prime} .
$$


In this case, denote by $H(P, Q)$ the Hopf invariant $H(\alpha(P), \alpha(Q)): S^{p-1} \longrightarrow \Sigma^{q} \Omega M$. When $q=0$ the relevant cofibration sequence is

$$
S^{p-1} \stackrel{\alpha(P)}{\longrightarrow} *=M^{\prime \prime} \longrightarrow S^{p}=M^{\prime \prime \prime} \hookrightarrow M
$$

and we let $H(P, Q)$ be the adjoint of the inclusion $S^{p} \hookrightarrow M$.

It is useful to notice here that to actually apply the constructions in 2.1 to construct $H(P, Q)$ we need to first replace $\alpha(P), \alpha(Q)$ by pointed maps. We do this as follows. First we fix a base point $\# \in Z(P, Q)$ and we consider in $M$ a path $\zeta \subset M$ that follows the flow $\gamma$ from $P$ to $Q$ through \# and then continues along a flow line of $\gamma$ from $Q$ to a distinguished point in $S^{q-1}=S^{u}(Q) \subset M^{\prime}$. Clearly, $\zeta$ is geometrically a broken flow line without self intersections and is therefore homeomorphic to the unit closed interval. We then replace $M$ and all our maps and spaces by those obtained after identifying the points belonging to $\zeta$ to a single point which is our choice of base-point. To avoid an unnecessarily complicated notation we continue to denote the maps and spaces obtained after this identification by the same symbols as before. We notice that our two cofibrations become pointed ones after this operation (in the sense that the maps are pointed and the attached cones are reduced).

Let

$$
K(P, Q)=\overline{W^{u}(P) \cap W^{s}(Q)} .
$$

This set is homeomorphic to the (un)reduced suspension $\Sigma Z(P, Q)$. Indeed, assuming that $a$ is a regular value of $f$, then $K(P, Q) \cup f^{-1}([a, \infty))$ consists of "segments" of gradient flow line of $f$ that start on $K(P, Q) \cap f^{-1}(a)$ and end in $P$. Therefore, $K(P, Q)$ is homeomorphic to the un-reduced cone over $K(P, Q) \cap f^{-1}(a) \approx Z(P, Q)$. A similar homeomorphism being valid for $K(P, Q) \cap f^{-1}(-\infty, a]$ the claim follows. We use the fixed base-point in $Z(P, Q)$ to construct an adjoint, $l(P, Q): Z(P, Q) \longrightarrow \Omega M$, of the inclusion $i_{Z}: \Sigma Z(P, Q) \hookrightarrow M$. It is also useful to note that this is coherent with identification of the path $\zeta$ to a single point as this identification transforms $K(P, Q)$ into a reduced suspension and the inclusion $\Sigma Z(P, Q) \rightarrow M$ becomes pointed.

By making the choices described in 2.2 we have a standard framing of the normal bundle of $Z(P, Q)$ in $S^{p-1} \approx S^{u}(P)$. Consider an inclusion of a tubular neighborhood

$$
N \approx D^{q} \times Z(P, Q) \subset S^{p-1}
$$

induced by this framing. We fix an orientation of $Z$ such that this inclusion is oriented. The Thom-Pontryagin construction applied to the fixed normal framing of $Z=Z(P, Q)$ together with the map $l(P, Q)$ gives the map

$$
\begin{aligned}
& T(P, Q): S^{p-1} \longrightarrow S^{p-1} / \overline{S^{p-1}-N}=\left(D^{q} \times Z\right) /\left(S^{q-1} \times Z\right) \stackrel{\Delta}{\longrightarrow} \\
& \quad\left(\left(D^{q} \times Z\right) /\left(S^{q-1} \times Z\right)\right) \wedge Z^{+} \stackrel{p_{1} \wedge l(P, Q)}{\longrightarrow} S^{q} \wedge(\Omega M)^{+}=S^{q} \vee S^{q} \wedge \Omega M .
\end{aligned}
$$

Here $\Delta$ is induced by the diagonal, $p_{1}$ is the projection on $S^{q}$ and we have again used the fact that $M$ is pointed. Recall that $h(P, Q)$ is the projection of $T(P, Q)$ on $S^{q} \wedge \Omega M$ and that the projection of $T(P, Q)$ on $S^{q}$ is $\delta_{f}(P, Q)$.

THEOREM 3.1. - The equality $\Sigma h(P, Q)=\Sigma H(P, Q)$ holds in $\pi_{p}\left(\Sigma^{q+1} \Omega M\right)$. In particular, $[Z(P, Q)]^{f r} \in \Omega_{p-q-1}^{f r}(\Omega M)$ equals the stable image of $H(P, Q)+\delta_{f}(P, Q)$.

Proof. - When $q=0$ the statement is immediate as $K(P, Q)=S^{p} \hookrightarrow M$. Assume from now on $q>0$. We put $Z=Z(P, Q)$. 
Notice that $h(P, Q)$ is also given by the composition

$$
S^{p-1} \stackrel{t}{\longrightarrow} S^{q} \wedge Z^{\Sigma^{q} l(P, Q)} \stackrel{\longrightarrow}{\longrightarrow} S^{q} \wedge \Omega M
$$

where

$$
t: S^{p-1} \longrightarrow\left(D^{q} \times Z\right) /\left(S^{q-1} \times Z\right) \rightarrow\left(D^{q} / S^{q-1}\right) \wedge Z^{+}=\left(S^{q} \times Z\right) /(* \times Z) \rightarrow S^{q} \wedge Z .
$$

The proof has five steps.

Some special neighborhoods of a critical point. As above, assume that for $\varepsilon>0$ sufficiently small, $P$ and $Q$ are the only critical points in $f^{-1}([f(Q)-\varepsilon, f(P)+\varepsilon])$. As before, let $M^{\prime}=f^{-1}(-\infty, f(Q)-\varepsilon]$ and $M^{\prime \prime}=f^{-1}(-\infty, f(P)-\varepsilon]$. Recall that $\gamma$ is the flow induced by $-\nabla f$ and let $D(x, r)$ be the closed disk in $M$ of radius $r$ and center $x$. Let

$$
\begin{gathered}
U_{\tau, \varepsilon}^{\prime}=\{x \in M: \\
\left.\gamma_{t}(Q) \in D(Q, \tau) \cap f^{-1}(f(Q))\right\} .
\end{gathered}
$$

It is useful to recall at this time that, by the Morse lemma, the topology of $f$ inside $U_{\tau, \varepsilon}^{\prime}$ is independent of $\tau$, for $\tau$ sufficiently small.

Let $U^{\prime}=U_{\tau, \varepsilon}^{\prime}$ for a small, fixed $\tau$. Then

$$
U^{\prime} \approx D^{q} \times D^{n-q}, \quad \partial U^{\prime}=A^{\prime} \cup B^{\prime} \cup C^{\prime}
$$

with

$$
\begin{aligned}
& S^{q-1} \times D^{n-q} \approx A^{\prime}=U^{\prime} \cap f^{-1}(f(Q)-\varepsilon), \\
& D^{q} \times S^{n-q-1} \approx C^{\prime}=U^{\prime} \cap f^{-1}(f(Q)+\varepsilon), \\
& B^{\prime} \approx S^{q-1} \times S^{n-q-1} \times[0,1]
\end{aligned}
$$

and $\nabla f$ is tangent to $B^{\prime}$ in all points $x \in \operatorname{Int}\left(B^{\prime}\right)$ (we consider the 0 -end of $B^{\prime}$ to be contained in $A^{\prime}$ ) and may be assumed to have flow lines of the form $\{a\} \times\{b\} \times[0,1]$ on $B^{\prime}$. Of course, $M^{\prime \prime} \simeq M^{\prime} \cup_{A^{\prime}} U^{\prime}$. It is obvious that there is a second neighborhood $U \subset U^{\prime}$ very close to $U^{\prime}$, homeomorphic to $U^{\prime}$, whose boundary admits the same structure as that of $U^{\prime}$ and such that if we denote by $A, B, C$ the respective pieces of the boundary of $U$, then $A=A^{\prime}, C \subset C^{\prime}, C \approx C^{\prime}$, $B \approx B^{\prime}$ and $\nabla f$ is transverse to $B$ and points inside $U$.

The attaching map $\alpha(P)$. We may define a deformation retract $r: M^{\prime \prime} \times[0,1] \longrightarrow M^{\prime \prime}$ whose 1-end $r_{1}$ sends each point in $M^{\prime \prime}$ along the flow $\gamma$ (induced by $-\nabla f$ ) to the point where it first reaches $M^{\prime} \cup_{A} U$. Let us denote $Z=Z(P, Q)$. Consider the inclusion $S^{p-1} \subset M^{\prime \prime}$ that represents the relative attaching map $\alpha(P)$. We see that $S^{p-1} \cap C \approx D^{q} \times Z$ (where we choose the framing of the respective tubular neighborhood of $Z$ in $S^{p-1}$ as described in 2.2). With this identification, we have

$$
\partial D^{q} \times Z=S^{q-1} \times Z \subset \partial C \approx S^{q-1} \times S^{n-q-1} .
$$

By using the deformation $r$ we may assume that the image of $\alpha(P)$ lies in $M^{\prime} \cup_{A} U$ and that $\alpha(P)\left(D^{q} \times Z, S^{q-1} \times Z\right) \subset(C, \partial C)$. Moreover, we may consider a collar neighborhood $V$ of $S^{q-1} \times Z$ in $S^{p-1}-\operatorname{Int}\left(D^{q} \times Z\right)$ such that

$$
V \approx S^{q-1} \times Z \times[0,1] \quad \text { with } S^{q-1} \times Z \times\{1\} \subset \partial D^{q} \times Z
$$


and with the property that (with the identifications described above)

$$
\left.\alpha(P)\right|_{V}: S^{q-1} \times Z \times[0,1] \longrightarrow B
$$

equals $\left.\alpha(P)\right|_{\left(S^{q-1} \times Z\right)} \times i d_{[0,1]}$ and $\alpha(P)\left(S^{p-1}-\left(V \cup D^{q} \times Z\right)\right) \subset M^{\prime}-A$.

Denote by $V^{\prime}=V \cup D^{q} \times Z, M^{*}=M^{\prime} \cup_{A} U$ and let $T=\overline{S^{p-1}-V^{\prime}}$. Of course, $V^{\prime} \approx D^{q} \times Z$.

We now intend to describe the composition

$$
c: S^{p-1} \stackrel{\alpha(P)}{\longrightarrow} M^{\prime \prime} \stackrel{\nabla}{\longrightarrow} S^{q} \vee M^{\prime \prime} \longrightarrow S^{q} \vee M .
$$

Here, $\nabla$ is the coaction and the last map is induced by inclusion. By the definition of $\nabla$ and making use of $r$ this map is homotopic to

$$
S^{p-1} \stackrel{\alpha(P)}{\longrightarrow} M^{\prime \prime} \stackrel{r_{1}}{\longrightarrow} M^{*} \stackrel{\nabla^{\prime}}{\longrightarrow} M^{*} / A \stackrel{w}{\longrightarrow} S^{q} \vee M^{*} \longrightarrow S^{q} \vee M
$$

Here $\nabla^{\prime}$ is the obvious collapsing map and the last map is, as before, the inclusion. The homotopy equivalence $w$ is the inverse of the obvious one obtained from the fact that $A \simeq S^{q-1}$ and using the standard flow induced null-homotopy of $A \hookrightarrow M^{*}$ (this is defined by first collapsing $A$ to its core $S^{q-1}=S^{u}(Q)$ and then collapsing this core to $Q$ along flow lines). In other words we have

$$
w: M^{*} / A \stackrel{i d}{\longrightarrow} U / A \vee M^{\prime} / A \stackrel{w^{\prime}}{\longrightarrow} S^{q} \vee M^{*}
$$

whith $w^{\prime}$ respecting the wedge, its restriction to $U / A$ is

$$
U / A=\left(D^{q} \times D^{n-q}\right) /\left(S^{q-1} \times D^{n-q}\right) \stackrel{p_{1}}{\longrightarrow} D^{q} / S^{q-1}=S^{q} .
$$

In the wedge $S^{q} \vee M^{*}$ the point $Q \in M^{*}$ is identified with the image of $S^{q-1}$ in the quotient $D^{q} / S^{q-1}$. The restriction of $w^{\prime}$ to $M^{\prime}$ sends $A$ to $Q$ by means of a map

$$
w^{\prime \prime}: M^{\prime} \longrightarrow M^{\prime} \cup W^{u}(Q) \hookrightarrow M^{*}
$$

which is the inclusion outside a neighborhood of $A$, is defined inside this neighborhood by using the null-homotopy mentioned above and induces a homotopy equivalence $M^{\prime} / A \longrightarrow M^{*}$.

Description of $c$. The map $c$ can be described by writing $S^{p-1}=V^{\prime} \cup_{\partial V^{\prime}} T$ and giving its restrictions to each of these two pieces. On $V^{\prime}$ the map $c$ is induced by the map of pairs $\left(V^{\prime} \approx D^{q} \times Z, S^{q-1} \times Z\right) \hookrightarrow(U, A)$ and composition with $w^{\prime}$; this sends $\partial T=\partial V^{\prime}=S^{q-1} \times Z$ to $Q$. On $T$ it is defined by $k=w^{\prime \prime} \circ r_{1} \circ \alpha(P)$.

We will now see that the composition $k^{\prime}: T \stackrel{k}{\longrightarrow} M^{*} \hookrightarrow M$ is homotopic $\operatorname{rel}(\partial T)$ to a map $k^{\prime \prime}$ defined as follows. Consider the inclusion of pairs $\left(D^{p}, S^{p-1}\right) \hookrightarrow\left(M, M^{\prime \prime}\right)$ whose restriction to $S^{p-1}$ is $\alpha(P)$ (here $D^{p} \subset W^{u}(P), D^{p} \cap M^{\prime \prime}=\partial D^{p}$ ). There is a deformation $d: D^{p} \longrightarrow M$ of this inclusion that is induced by the flow $\gamma$, collapses $Z \subset S^{p-1}$ to $Q$ along $\gamma$, is constant ouside a neighborhood of $Z$ in $D^{p}$ and factors as $D^{p} \longrightarrow D^{p} \cup W^{s}(Q) \hookrightarrow M$. As $D^{p} \cup\left(W^{s}(Q) \cap M^{\prime \prime}\right) \simeq \Sigma Z$ that means that, up to homotopy, $d$ factors through $i_{Z}$. In $S^{p-1}$ there is a deformation $l: T \longrightarrow S^{p-1}$ that is constant outside a neighborhood of $\partial T$ and that sends the point $(x, y) \in S^{q-1} \times Z=\partial T$ to $(0, y) \in Z$. The map $k^{\prime \prime}$ is given by $i_{Z} \circ d \circ l$. To see that $k^{\prime}$ and $k^{\prime \prime}$ are homotopic, notice that they are both homotopic (rel boundary) to the following 
map $k^{\prime \prime \prime}:$ transport $T$ homeomorphically along the flow till it reaches $f^{-1}(f(Q))$. Let $T^{\prime}$ be the image of $T$ inside this singular hypersurface. Use the conical structure of $f^{-1}(f(Q))$ around $Q$ to deform $\partial T^{\prime}$ to $Q$ without leaving $f^{-1}(f(Q))$ and whithout moving points that are outside a small neighborhood of $\partial T^{\prime}$.

It follows that we may use the map $k^{\prime \prime}$ instead of $k^{\prime}$ in the description of $c$. We get the following commutative diagram:

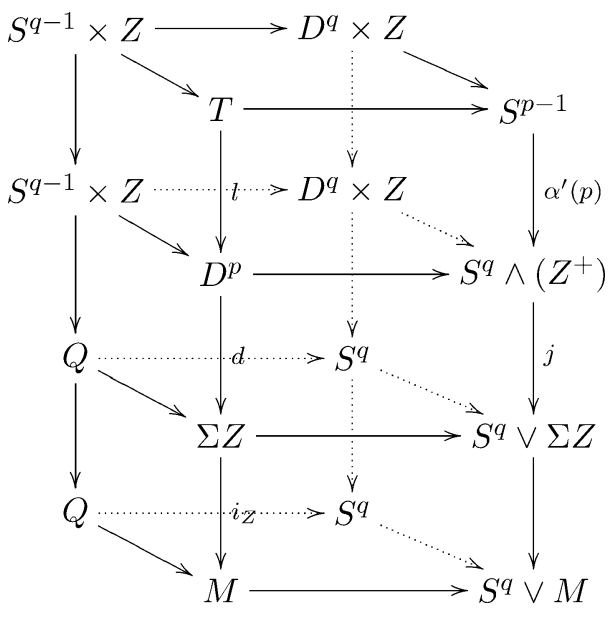

In this diagram all horizontal squares are push outs and the vertical maps in the right corner are induced by the other three. We have identified $V^{\prime}$ to $D^{q} \times Z$. The composition originating in $T$ is the map $k^{\prime \prime}$ described above and that defined on $D^{q} \times Z$ is the restriction of $c$ (which, as mentioned above is the projection on $D^{q}$ followed by the collapsing to $D^{q} / S^{q-1}=S^{q}$ ). Therefore, the composition in the right corner is homotopic to $c$. After one suspension, the map $\alpha^{\prime}(P)$ is additive with respect to the connected components of $Z$.

Identification of a Whitehead product. The next step is to consider the map

$$
j: S^{q} \vee S^{q} \wedge Z \longrightarrow S^{q} \vee \Sigma Z
$$

of the diagram above (where we use $S^{q} \wedge\left(Z^{+}\right)=S^{q} \vee S^{q-1} * Z$ ) and show that its restriction to $S^{q-1} * Z=S^{q} \wedge Z$ is homotopic to the (generalized) Whitehead product of the inclusions $S^{q} \hookrightarrow S^{q} \vee \Sigma Z$ and $\Sigma Z \hookrightarrow S^{q} \vee \Sigma Z$. For this consider the next commutative diagram.

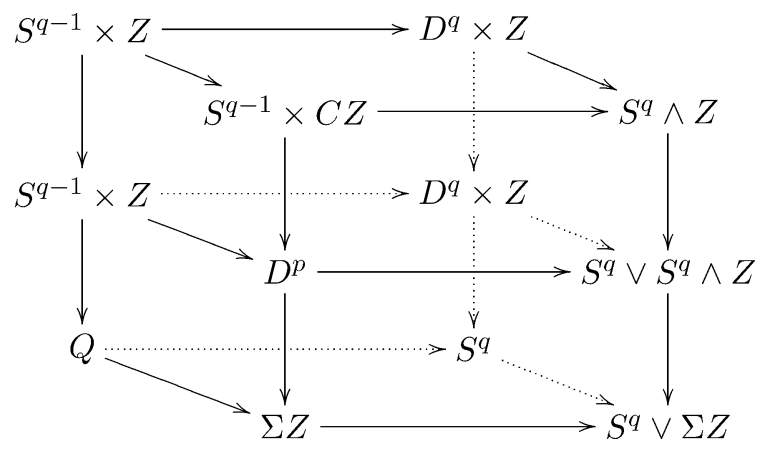


Again, the horizontal squares are push outs and the vertical maps in the right corner are induced by the respective three others, $C Z$ is the cone on $Z$. The map $S^{q-1} \times Z \longrightarrow D^{p}$ which is the restriction of $l$ to $\partial T$ factors as $S^{q-1} \times Z \subset S^{q-1} \times C Z \stackrel{p_{2}}{\longrightarrow} C Z \hookrightarrow D^{p}$ (the last map being the inclusion of $C Z$ in $W^{u}(P)$, the vertex of this cone being identified to $P$ ) and it is this factorization that is used in the upper, vertical, left square. It is clear that the top, vertical map in the right corner is, up to homotopy, the inclusion on the first factor. Now, the composition $S^{q-1} \times C Z \longrightarrow D^{p} \longrightarrow \Sigma Z$ is given by projection onto $C Z$ and collapsing onto $\Sigma Z$. Similarly, $D^{q} \times Z \longrightarrow S^{q}$ is projection onto $D^{q}$ and then collapsing onto $S^{q}$. This shows, by the definition of the generalized Whitehead product as it appears in [3] (see also [40] for the classical case of the wedge of two spheres), that the composition in the right corner is the wanted Whitehead product.

We therefore obtain that $c$ is homotopic to the composition

$$
c^{\prime}: S^{p-1} \stackrel{\alpha^{\prime}(P)}{\longrightarrow} S^{q} \vee S^{q} \wedge Z \stackrel{h}{\longrightarrow} S^{q} \vee \Sigma Z \stackrel{i d \vee i_{Z}}{\longrightarrow} S^{q} \vee M
$$

with $h=i d_{S^{q}} \vee\left[i_{S^{q}}, i_{\Sigma Z}\right]$.

Identification of the Hopf invariant. The last step of the proof is to use the factorization of $c^{\prime}$ to evaluate the relevant Hopf invariant. We now look to $\Omega(h)$ and use the standard splitting of the loop space of a wedge to write the Hopf invariant $H(P, Q)$ as the adjoint of the composition

$$
\begin{aligned}
h^{\prime}: S^{p-2} \stackrel{i}{\longrightarrow} \Omega S^{p-1} & \stackrel{u}{\longrightarrow} \Omega\left(S^{q} \wedge Z\right) \times \Omega\left(\Omega S^{q} * \Omega\left(S^{q} \wedge Z\right)\right) \\
& \stackrel{v}{\longrightarrow} \Omega\left(\Omega S^{q} * \Omega \Sigma Z\right) \stackrel{y}{\longrightarrow} \Omega\left(S^{q} \wedge \Omega \Sigma Z\right) \stackrel{i_{Z}}{\longrightarrow} \Omega\left(S^{q} \wedge \Omega M\right) .
\end{aligned}
$$

This shows that the Hopf invariant verifies $H(P, Q) \simeq \phi+\phi^{\prime}$ where $\phi$ is the adjoint of $i_{Z} \circ y \circ v \circ i_{1} \circ p_{1} \circ u \circ i$ and similarly $\phi^{\prime}$ is the adjoint of $i_{Z} \circ y \circ v \circ i_{2} \circ p_{2} \circ u \circ i$ where $i_{k}, p_{k}$ are the respective inclusions and projections of the two factors in the product $\Omega\left(S^{q} \wedge Z\right) \times \Omega\left(\Omega S^{q} * \Omega\left(S^{q} \wedge Z\right)\right)$. This is due to the fact that the suspension of a map $a: \Sigma T \rightarrow S \times S^{\prime}$ equals $\Sigma\left(p_{1} \circ a\right)+\Sigma\left(p_{2} \circ a\right)$ and to the fact that the adjoint of a map $b: T \rightarrow \Omega S$ is homotopic to $e v \circ \Sigma b$ where $e v: \Sigma \Omega S \rightarrow S$ is the evaluation. Another useful fact is that the generalized (universal) Whitehead product $\Sigma\left(S \wedge S^{\prime}\right) \stackrel{\left[i_{1}, i_{2}\right]}{\longrightarrow} \Sigma S \vee \Sigma S^{\prime}$ is homotopic to the composition

$$
\Sigma\left(S \wedge S^{\prime}\right) \stackrel{\Sigma\left(e_{1} \wedge e_{2}\right)}{\longrightarrow} \Sigma\left(\Omega \Sigma S \wedge \Omega \Sigma S^{\prime}\right)=\Omega \Sigma S * \Omega \Sigma S^{\prime} \longrightarrow \Sigma S \vee \Sigma S^{\prime}
$$

where $e_{i}$ are adjoints of the respective identities and the last map is the inclusion of the homotopy fibre of $\Sigma S \vee \Sigma S^{\prime} \rightarrow \Sigma S \times \Sigma S^{\prime}$ [13]. Applying this property to $S=S^{q-1}$ and $S^{\prime}=Z$ we see that the restriction of $h^{\prime \prime}=y \circ v$ to $\Omega\left(S^{q} \wedge Z\right)$ is just the looping of the map $S^{q} \wedge Z \longrightarrow S^{q} \wedge \Omega \Sigma Z$ induced by the inclusion $Z \hookrightarrow \Omega \Sigma Z$. The projection of the map $\alpha^{\prime}(P)$ on $S^{q} \wedge Z$ coincides by construction with the map $t$. Therefore, $\phi$ is given by the composition

$$
S^{p-1} \stackrel{t}{\longrightarrow} S^{q} \wedge Z \longrightarrow S^{q} \wedge \Omega \Sigma Z \longrightarrow S^{q} \wedge \Omega M
$$

where the composition $S^{q} \wedge Z \longrightarrow S^{q} \wedge \Omega \Sigma Z \longrightarrow S^{q} \wedge \Omega M$ is the qth suspension of the adjunction of the inclusion $i_{Z}$. Thus $\phi=h(P, Q)$.

We now use the diagram below to identify the second homotopy class, $\phi^{\prime}$, with the composition $S^{p-1} \stackrel{s}{\longrightarrow} \Omega S^{q} * \Omega\left(S^{q} \wedge Z\right) \stackrel{z}{\longrightarrow} \Omega S^{q} * \Omega \Sigma Z \longrightarrow S^{q} \wedge \Omega M$ where $s$ is the adjunction of $p_{2} \circ u \circ i$ 
and $z$ is the top composition in the diagram.

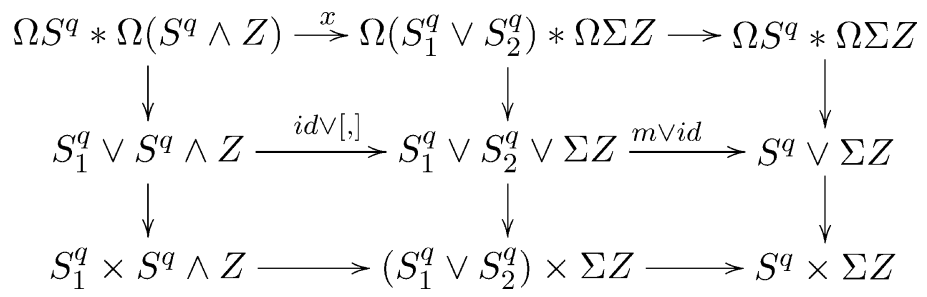

Here, the columns are fibrations and the top row is induced by the bottom two; $m$ is the folding map of the two spheres $S_{1}^{q}$ and $S_{2}^{q}$; the bottom, left, horizontal map is trivial when projected on $\Sigma Z$ and factors via the inclusion of $S_{1}^{q}$.

We now compose $z$ with the evaluation $e v: \Sigma \Omega S^{q} \wedge \Omega \Sigma Z \longrightarrow S^{q} \wedge \Omega \Sigma Z$. The composition $e v \circ z$ factors via the evaluation

$$
e v^{\prime}: \Sigma \Omega\left(S_{1}^{q} \vee S_{2}^{q}\right) \wedge \Omega \Sigma Z \longrightarrow\left(S_{1}^{q} \vee S_{2}^{q}\right) \wedge \Omega \Sigma Z=\left(S_{1}^{q} \wedge \Omega \Sigma Z\right) \vee\left(S_{2}^{q} \wedge \Omega \Sigma Z\right) .
$$

Notice that the projection of $e v^{\prime} \circ x$ on each of the factors of the wedge is null. This implies that $\Sigma \phi^{\prime}=0$ and concludes the proof.

Remark 4. - It is instructive to consider the case of the Morse-Smale function

$$
f: S^{2} \times S^{2} \longrightarrow \mathbf{R}
$$

with precisely four critical points. Denote by $P$ the maximum and let $Q$ be one of critical points of index two. The equality $\Sigma H(P, Q)=\Sigma h(P, Q)$ comes down to the fact that the ThomPontryagin construction applied disjointly to two circles embedded with linking number one and trivially framed in $S^{3}$ produces the Whitehead product $S^{3} \longrightarrow S^{2} \vee S^{2}$.

Here is a first context in which $h(P, Q)$ is relevant. Suppose $f, P, Q$ are as in the theorem and fix a fiber bundle on $M$ that is classified by a map $\mu: M \longrightarrow \mathbf{B S O}(m)$ with $p-2 q<m$. For $x \in \Omega_{*}^{f r}(\Omega M)$ let $\bar{\mu}(x) \in \Omega_{*}^{f r}(\Omega M)$ be defined as follows. Consider $X \stackrel{g}{\longrightarrow} \Omega M$ together with an embedding $X \stackrel{i}{\hookrightarrow} S^{k}$ and a normal framing representing $x$. The class $\bar{\mu}(x)$ is represented by $X \stackrel{g}{\longrightarrow} \Omega M$ together with a normal framing

$$
X \stackrel{i}{\hookrightarrow} S^{k} \stackrel{j}{\hookrightarrow} S^{k+m}
$$

given at a point $a \in X$ by $\left((\Omega \mu \circ g)(a)\left(i^{*}(j)_{a}\right), i\right)$ (here $j$ is the standard normal framing of $S^{k}$ in $S^{k+m}$ ).

In a tubular neighborhood $Y$ of $M$ in the total space of $\mu$ we may consider a function $g: Y \longrightarrow \mathbf{R}$ giving the square of the distance from $M$. If $Y$ is sufficiently small this function is non-degenerate in the direction of the fibre and, in particular, the difference $f^{\prime}=f \circ p-g$ is Morse (here $p: Y \longrightarrow M$ is the restriction of the projection of the bundle). The critical points $P$ and $Q$ are again consecutive non-degenerate critical points of $f^{\prime}$. Their indexes are respectively $m+p$ and $m+q$. Let $Z^{\mu}(P, Q)$ be the (framed) connecting manifold of $f^{\prime}$ (as a space it coincides with $Z(P, Q)$ but its framing might be different). Recall that $\delta_{f}(P, Q)^{\mu}$ is the relative attaching map induced on the Thom space of $\mu$ by $\delta_{f}(P, Q)$. It is easy to see [6] that $\delta_{f^{\prime}}(P, Q)=\delta_{f}(P, Q)^{\mu}$.

For $x \in \pi_{k+n}\left(S^{n}\right)$ we denote by $\{x\} \in \pi_{k}^{S}$ its stable image. 
PROPOSITION 3.2. - In $\Omega_{p-q-1}^{f r}(\Omega M)$ we have $\left[Z^{\mu}(P, Q)\right]^{f r}=\bar{\mu}\left([Z(P, Q)]^{f r}\right)$. Moreover, if $h^{*}(P, Q) \in \pi_{p-q-1} \Omega^{q} \Sigma^{q} \Omega M$ denotes the qth order adjoint of $h(P, Q)$, then the stable difference $\left\{\delta_{f}(P, Q)^{\mu}\right\}-\left\{\delta_{f}(P, Q)\right\}$ equals

$$
J^{q}\left(\Omega^{q} \Sigma^{q}(\Omega \mu) \circ h^{*}(P, Q)\right) .
$$

Proof. - We use the notations fixed in the proof of the theorem. As seen above, the bordism class of $Z(P, Q)$ in $\Omega M$ is given by a map

$$
S^{p-1} \stackrel{\alpha}{\longrightarrow} S^{q} \wedge Z^{+} \stackrel{i d \wedge l(P, Q)}{\longrightarrow} S^{q} \wedge(\Omega M)^{+} .
$$

Of course, the bordism class of $Z^{\mu}(P, Q)$ is given by a similar map

$$
S^{m+p-1} \stackrel{\alpha^{\prime}}{\longrightarrow} S^{m+q} \wedge Z^{+} \stackrel{i d \wedge l(P, Q)}{\longrightarrow} S^{m+q} \wedge(\Omega M)^{+} .
$$

In general, the map $\alpha^{\prime}$ is not the suspension of $\alpha$. It is immediate that, as in [7], $\alpha^{\prime}=e \circ \Sigma^{m} \alpha$ where $e: S^{m+q} \wedge Z^{+} \longrightarrow S^{m+q} \wedge Z^{+}$is a homotopy equivalence that appears at the passage (along the flow $\gamma_{1}$ induced by $-\nabla f^{\prime}$ ) from a neighborhood of $P$ to one of $Q$. More precisely $e$ is induced by the map of pairs

$$
e^{\prime}:\left(D^{m} \times D^{q} \times Z, S^{m-1} \times D^{q} \times Z\right) \longrightarrow\left(D^{m} \times D^{q} \times Z, S^{m-1} \times D^{q} \times Z\right)
$$

that takes $(x, y, z)$ to $\left(\mu^{*}(z)(x), y, z\right)$ where $\mu^{*}: Z \stackrel{l(P, Q)}{\longrightarrow} \Omega M \stackrel{\Omega \mu}{\longrightarrow} \mathbf{S O}(m)$. The map $e^{\prime}$ induces $e$ by collapsing $S^{m+q-1} \times Z$ to a point. The first part of the statement is now clear. Moreover, notice that $p_{1} \circ e$ restricted to the $q+m$ skeleton (which is a wedge of $(q+m)$-dimensional spheres in number equal to the number of connected components of $Z$ ) is the identity because the bundle is oriented. Its restriction to $S^{q+m} \wedge Z$ is $\Sigma^{q-1} J\left(\mu^{*}\right)$. Here, $J\left(\mu^{*}\right): S^{m} \wedge Z \longrightarrow S^{m}$ is defined by the Hopf construction on the map $S^{m-1} \times Z \longrightarrow S^{m-1}$ given by $(x, y) \longrightarrow \mu^{*}(y)(x)$. Because $p-2 q<m, \delta_{f^{\prime}}(P, Q)=\Sigma^{m} \delta_{f}(P, Q)+\Sigma^{q-1} J\left(\mu^{*}\right) \circ t$. The statement follows from the definition of $J^{q}$.

Remark 5. - (a) It is clear that, as $\Sigma h(P, Q)=\Sigma H(P, Q)$, we may replace in the formula above $h(P, Q)$ by $H(P, Q)$. When $p>q+1$ notice also that $[h(P, Q)]=[H(P, Q]$ when viewed in $H_{p-q-1}(\Omega M)$. Moreover, in this case, $[h(P, Q)]$ is the fundamental class of $Z(P, Q)$ in $\Omega M$.

(b) The second part of the above proposition, with $H(P, Q)$ in the place of $h(P, Q)$, is also a consequence of purely homotopical results of Dula [9].

(c) The proof of the proposition is in fact the direct specialization to the Morse-Smale case of a result established in [7] for reasonable critical points (a class that contains all isolated, analytic singularities).

For the next result we consider two critical points $P$ and $R$ of $f$ (which is a function as before) such that $P$ and $R$ are not necessarily consecutive but $f(P)>f(R)$ and if $Q$ is a critical point such that $Q \in \overline{W^{u}(P)} \cap \overline{W^{s}(R)}$, then $P$ and $Q$ are consecutive and so are $Q$ and $R$ (in other words all broken conecting flow lines between $P$ and $R$ are broken in just one point). We denote by $I(P, R)$ the set of all such intermediate critical points $Q$ associated to $P$ and $R$.

For $P$ and $Q$ consecutive of indexes respectively $p$ and $q$ recall that we denote by $[Z(P, Q)]^{f r} \in \Omega_{p-q-1}^{f r}(\Omega M)$ the ambient bordism class of $Z(P, Q)$. We assume that $M$ is simply connected (which implies that the various bordism classes involved are independent of choices of base-points), oriented and we make the choice of orientations described in 2.2. As before, 
assume the connecting manifolds $Z(P, Q)$ oriented such that the standard framing inside $S^{p-1}$ summed with this orientation gives the standard orientation of $S^{p-1}$.

THEOREM 3.3. - With the assumptions above we have:

$$
\sum_{Q \in I(P, R)}(-1)^{(p+r) q}[Z(P, Q)]^{f r} \bullet[Z(Q, R)]^{f r}=0
$$

where $\bullet$ is the product in $\Omega_{*}^{f r}(\Omega M)$.

Proof. - We may assume whithout loss of generality that $I(P, R)$ is contained is the same critical level $f^{-1}(c)$. Let

$$
K(P, R)=\left\{x \in M: \lim _{t \rightarrow+\infty}\left(\gamma_{t}(x)\right)=R, \lim _{t \rightarrow-\infty}\left(\gamma_{t}(x)\right)=P\right\} \cup\{P, R\} .
$$

The proof is based on understanding how the ends of $K(P, R)$ are embedded in $M$.

Identification of the ends of $K(P, R)$. Let $p, r, q$ be repectively the indexes of $P, R$ and $Q \in I(P, R)$. Consider $a \in \mathbf{R}$ such that $f(R)<a<f(P)$ and let $Z(P, R)=f^{-1}(a) \cap K(P, R)$. Then $Z(P, R)$ is a manifold of dimension $p-r-1$ (of course, $Z(P, R)$ is not closed) and its homeomorphism type does not depend on the choice of $a$. Clearly, $K(P, R)$ is homeomorphic to the unreduced suspension of $Z(P, R)$. We also recall the notation $K(P, Q)=\overline{W^{u}(P) \cap W^{s}(Q)}$.

Around each critical point in $I(P, R)$ we assume fixed a Morse chart inside which the metric is the canonical one. Let $K_{\tau}(P, R)$ be the set of all points $x \in K(P, R)$ such that if for some $t \in \mathbf{R}$ we have $\gamma_{t}(x) \in f^{-1}(c)$, then $d\left(\gamma_{t}(x), Q\right) \geqslant \tau$ for all $Q \in I(P, R)(d($,$) being the distance in$ $M)$. For $\tau$ sufficiently small this set is the (unreduced) suspension over

$$
Z_{\tau}(P, R)=\left(K(P, R) \cap f^{-1}(c)\right)-\bigcup_{Q \in I(P, R)} D(Q, \tau)
$$

where $D(Q, \tau)$ is the disk in $M$ of center $Q$ and of radius $\tau$ (the intersection of $S(Q, \tau)=$ $\partial D(Q, \tau)$ and $f^{-1}(c)$ is certainly transverse for $\tau$ small enough).

Notice that $Z_{\tau}(P, R)$ is a manifold with boundary whose homeomorphism type does not depend on the choice of $\tau$, if this constant is smaller than some fixed $\tau^{\prime}>0$, and that its interior is homeomorphic to $K(P, R)$. These statements follow from the Morse-Smale condition. Indeed, for $Q \in I(P, R)$ let $G(Q, \tau)=f^{-1}(c) \cap S(Q, \tau)$. Clearly, $G(Q, \tau) \approx S^{q-1} \times S^{n-q-1}$. Consider a neighborhood of $Q$ as described at the beginning of the proof of 3.1, $U^{\prime}=U_{\tau, \varepsilon}^{\prime}$ and recall that for small $\varepsilon$ and $\tau, C^{\prime}=\partial U^{\prime} \cap f^{-1}(c+\varepsilon)=D^{q} \times S^{n-q-1}$ and with this identification $\{0\} \times S^{n-q-1}=S^{s}(Q)$. The intersection of $W^{u}(P)$ with $f^{-1}(c+\varepsilon)$ is identified with $S^{u}(P)$ and therefore it intersects transversely $S^{s}(Q)$ as well as $\partial C^{\prime}$. This implies immediately that $W^{u}(P)$ intersects transversely $G(Q, \tau)$. By the same method we obtain that $W^{s}(R)$ intersects transversely $G(Q, \tau)$. This shows that for all small enough $\tau$ the intersection of $K(P, Q)$ and $G(Q, \tau)$ is transverse. This implies all the claimed properties of $Z_{\tau}(P, R)$.

Fix $Q$ and some $\tau$ as above and let

$$
\begin{aligned}
& \partial_{Q} Z=\partial Z_{\tau}(P, R) \cap S(Q, \tau), \\
& \partial_{Q} K=\left\{x \in K_{\tau}(P, R): \exists t, \gamma_{t}(x) \in S(Q, \tau) \cap f^{-1}(c)\right\} \cup\{P, R\} .
\end{aligned}
$$

Clearly, $\partial_{Q} K$ is the suspension over $\partial_{Q} Z$ and, in particular, if for $a \in \mathbf{R}$ such that $f(R)<a<f(P)$ we denote $\partial_{Q}^{a}=\partial_{Q} K \cap f^{-1}(a)$, then $\partial_{Q}^{a}$ is homeomorphic to $\partial_{Q} Z$. 
Embedding of $\partial_{Q} K$ in $M$. Let

$$
W(P, Q)=\left\{x \in W^{u}(P): f(x) \geqslant c, \exists t \gamma_{t}(x) \in f^{-1}(c), d\left(\gamma_{t}(x), Q\right) \leqslant \tau\right\} \cup K(P, Q)
$$

and

$$
W(R, Q)=\left\{x \in W^{s}(R): f(x) \leqslant c, \exists t \gamma_{t}(x) \in f^{-1}(c), d\left(\gamma_{t}(x), Q\right) \leqslant \tau\right\} \cup K(Q, R) .
$$

Let $a$ and $b$ be such that $f(R)<b<c<a<f(P)$. Clearly, the interiors of $W(P, Q)$ and $W(R, Q)$ are (open) cones over their intersection with $f^{-1}(a)$ and respectively $f^{-1}(b)$. In particular, we have

$$
W_{a}(P)=W(P, Q) \cap f^{-1}(a) \approx D^{q} \times Z(P, Q)
$$

and

$$
W_{b}(R)=W(R, Q) \cap f^{-1}(b) \approx Z(Q, R) \times D^{n-q} .
$$

The set $H(Q, \tau)=\left\{x \in f^{-1}(c): d(x, Q) \leqslant \tau\right\}$ is clearly a cone over its boundary which is $G(Q, \tau)$. Also $\partial_{Q}^{a} \subset W_{a}(P)$ and $\partial_{Q}^{b} \subset W_{b}(R)$.

Notice, that the union $W(P, Q) \cup W(R, Q) \cup H(Q, \tau)$ has the homotopy type of the wedge $\Sigma Z(P, Q) \vee \Sigma Z(Q, R)$ and $H(Q, \tau) \cup \partial_{Q} K \simeq \Sigma \partial_{Q} Z \vee \Sigma \partial_{Q} Z$. Therefore the inclusion

$$
\partial_{Q} K \longrightarrow H(Q, \tau) \cup \partial_{Q} K \longrightarrow W(P, Q) \cup W(R, Q) \cup H(Q, \tau) \longrightarrow M
$$

is seen to be homotopic to

$$
\partial_{Q} K=\Sigma \partial_{Q} Z \longrightarrow \Sigma \partial_{Q} Z \vee \Sigma \partial_{Q} Z \longrightarrow \Sigma Z(P, Q) \vee \Sigma Z(Q, R) \longrightarrow M
$$

where the first map is the pinch map, the second is given by wedging the suspensions of

$$
t_{1}: \partial_{Q}^{a} \subset W_{a}(P)=D^{q} \times Z(P, Q) \stackrel{p_{2}}{\longrightarrow} Z(P, Q)
$$

and

$$
t_{2}: \partial_{Q}^{a^{\prime}} \subset W_{a^{\prime}}(R)=Z(Q, R) \times D^{n-q} \stackrel{p_{1}}{\longrightarrow} Z(Q, R)
$$

and the third map is the inclusion of $K(P, Q) \vee K(Q, R)$.

Because $M$ is simply connected we have a well defined map $l(P, R): \partial_{Q} Z \longrightarrow \Omega M$ which is the adjunct of the inclusion $\partial_{Q} K \subset M$. From the factorization above, by adjunction, we obtain that $l(P, R)$ factors as

$$
\partial_{Q} Z \longrightarrow Z(P, Q) \times Z(Q, R) \longrightarrow \Omega M \times \Omega M \longrightarrow \Omega M
$$

where the first map is the product $t_{1} \times t_{2}$, the second map is the product of the adjoints of the respective inclusions and the third is the loop product.

Description of $\partial_{Q} Z$. We want to observe that $t_{1} \times t_{2}$ is a homeomorphism. Fix $a$ and $b$ such that $f(R)<b \leqslant c \leqslant a<f(P)$ and $c-b<\varepsilon, a-c<\varepsilon$. We have the homeomorphsims $\partial_{Q}^{b} \approx \partial_{Q} Z \approx \partial_{Q}^{a}$ induced by transporting the respective sets along the flow $\gamma$. Consider the inclusion $\partial_{Q}^{a} \hookrightarrow S^{q-1} \times Z(P, Q)$ here

$$
S^{q-1} \times Z(P, Q) \subset D^{q} \times Z(P, Q)=W^{u}(P) \cap f^{-1}(a) \cap U^{\prime} .
$$


By transporting it along the flow $\gamma$ till we reach the level set $f^{-1}(b)$ we obtain an inclusion

$$
\partial_{Q}^{b} \hookrightarrow S^{q-1} \times Z(P, Q) \stackrel{j}{\hookrightarrow} S^{q-1} \times S^{n-q-1}=\partial U^{\prime} \cap f^{-1}(b)
$$

(notice that now $S^{q-1}$ is identified here to $S^{u}(Q)$ ). The map $j$ is the identity on the first factor and the usual inclusion on the second. At the same time we also have an inclusion $\partial_{Q}^{b} \hookrightarrow Z(Q, R) \times S^{n-q-1}$. It follows that the product $t_{2} \times t_{1}$ is identified to the inclusion

$$
v: \partial_{Q}^{b} \hookrightarrow Z(Q, R) \times Z(P, Q)=\left(Z(Q, R) \times S^{n-q-1}\right) \cap\left(S^{q-1} \times Z(P, Q)\right) .
$$

On the other hand each point of this last intersection belongs to $\partial_{Q}^{b}$. Indeed, with the given parametrizations all the points in $S^{q-1} \times Z(P, Q)$ belong to $W^{u}(P)$ and all the points in $Z(Q, R) \times S^{n-q-1}$ belong to $W^{s}(R)$. Therefore $v$ is also surjective.

Consequences in $\Omega_{*}^{f r}(\Omega M)$. Consider the framing of $\partial_{Q} Z=Z(P, Q) \times Z(Q, R)$ inside $S^{n-q-1} \times S^{q-1}$ obtained as the restriction of the normal framing of $K(P, R)$ inside $f^{-1}(a)$. This framing is the product of the framing induced by the standard framing of $S^{p-1}$ with that induced by the standard framing of $S^{n-r-1}$. Therefore, the framing of $Z(P, Q) \times Z(Q, R)$ inside $S^{n-q-1} \times S^{q-1}$ coincides with the product of the standard framings of $Z(P, Q)$ in $S^{n-q-1}$ (induced by the framing of $\left.S^{p-1}\right)$ and the standard framing of $Z(Q, R)$ in $S^{q-1}$. Let $\bar{Z}(P, Q)$ be the framed bordism representative given by $l(P, Q)$ and the framing of $Z(P, Q)$ inside $S^{n-q-1}$. The orientation of $\bar{Z}(P, Q)$ is such that the framing summed with this orientation gives the standard orientation on $S^{n-q-1}$.

We orient $Z(P, R)$ such that the ordered sum of the framings induced first from $S^{p-1}$, from $S^{n-r-1}$ and the orientation of $Z(P, R)$ gives the orientation of $f^{-1}(a)$.

Assume now that $M$ has trivial stable normal bundle. In this case, after embedding $M$ in a high-dimensional sphere we see that because of (1) and as

$$
\coprod_{Q \in I(P, R)} \partial_{Q} Z=\partial\left(Z_{\tau}(P, R)\right)
$$

we have $\Sigma_{Q \in I(P, R)} \varepsilon^{\prime}(Q)[\bar{Z}(P, Q)]^{f r} \bullet[Z(Q, R)]^{f r}=0$.

The sign $\varepsilon^{\prime}(Q)$ is +1 if the orientation induced from that of $Z(P, R)$ on $\bar{Z}(P, Q) \times Z(Q, R)$ coincides with the product orientation and is -1 otherwise. With our conventions $\varepsilon^{\prime}(Q)=$ $(-1)^{(n-r) q} \varepsilon_{0}$ where $\varepsilon_{0}$ depends only on $p, r, n$.

We now return to the case when the stable bundle of $M$ is general. Similarly to the proof of Proposition 3.2 we consider a function $f^{\prime}=f \circ p+g$ defined on a neighborhood $U$ of the 0 -section of the normal bundle $\nu$ of an embedding of $M$ in a high dimensional sphere $S^{n+m}$. Here, $g$ is the square of a distance function measuring the distance from $M$. We use the notation $Z^{-\nu}(P, Q)$ for the connecting manifold of $P$ and $Q$ for the function $f^{\prime}$. By the formula above we have

$$
\Sigma_{Q \in I(P, R)}(-1)^{(m+n-r) q}\left[\overline{Z^{-\nu}}(P, Q)\right]^{f r} \bullet\left[Z^{-\nu}(Q, R)\right]^{f r}=0 .
$$

We have

$$
\left[Z^{-\nu}(Q, R)\right]^{f r}=[Z(Q, R)]^{f r}, \quad\left[Z^{-\nu}(P, Q)\right]^{f r}=[Z(P, Q)]^{f r}
$$

Moreover, because the stable normal bundle of $U$ is trivial, the framings of $\overline{Z^{-\nu}}(P, Q)$ and $Z^{-\nu}(P, Q)$ are the same up to a sign coming from a possible difference in orientations. With our conventions the sign is $(-1)^{(m+n-p) q}$. 
Remark 6. - In [4,5] Cohen, Jones and Segal pursue a systematic analysis of the ends of the moduli spaces of connecting flow lines (even for non-consecutive critical points) together with the relevant framings (the definition they use for these framings is somewhat different from ours, though). In particular, the fact that $t_{1} \times t_{2}$ above is a homeomorphism is a direct consequence of a more general result of Betz as described in [4]. For completeness we have included a direct justification in the proof above.

\section{Applications and examples}

As in the last section $M^{n}$ is a compact, smooth, riemannian, connected manifold. Suppose $f: M \longrightarrow \mathbf{R}$ is a Morse-Smale function and $P$ and $Q$ are consecutive critical points of index, respectively, $p$ and $q$. Recall that $h^{*}(P, Q) \in \pi_{p-q-1} \Omega^{q} \Sigma^{q} \Omega M$ is the $q$ th order adjoint of $h(P, Q)$. Let $h^{S}(P, Q) \in \pi_{p-q-1}^{S}(\Omega M)$ be the class of $h^{*}(P, Q)$. Let

$$
k(P, Q)=\min \left\{j: h^{S}(P, Q) \in \operatorname{Im}\left(\Omega^{j} \Sigma^{j} \Omega M\right)\right\} .
$$

This gives a measure of the embedding complexity of $Z(P, Q)$. Indeed, if the framed embedding $Z(P, Q) \hookrightarrow S^{p-1}=S^{u}(P)$ factors as a composition of framed embeddings $Z(P, Q) \hookrightarrow S^{t-1} \hookrightarrow S^{p-1}$, then $k(P, Q) \leqslant t-p+q$. For example, if $k(P, Q)>0$, then $Z(P, Q)$ is not a sphere.

Of course, as $k(P, Q)$ is defined homotopically, it is invariant to deformations of $f$ via MorseSmale functions.

We intend here to give a method to estimate $k(P, Q)$. In particular, we construct examples when $k(P, Q)=\operatorname{ind}(Q)$ even if $\delta_{f}(P, Q)=0$. Of course, we always have $k(P, Q) \leqslant \operatorname{ind}(Q)$. Along the way, we also detect certain Poincaré complexes that are not smoothable.

\subsection{Non-smoothable Poincaré duality complexes and embedding complexity of connecting manifolds}

We start with two simple consequences of 3.2.

Corollary 4.1. - Assume that $f: M \longrightarrow \mathbf{R}$ is a smooth Morse-Smale function and that $P$ and $Q$ are consecutive critical points of $f$. Then, for some $\varepsilon \in\{-1,1\}$, we have

$$
\varepsilon\left\{\delta_{-f}(Q, P)\right\}=\left\{\delta_{f}(P, Q)\right\}+\Delta(P, Q),
$$

where $\{x\}$ is the stable image of $x$ and $\Delta(P, Q)=J^{q}\left(\Omega^{q} \Sigma^{q}(\Omega \nu) \circ h^{*}(P, Q)\right)$ ( $\nu$ is the stable normal bundle of $M$ ).

Proof. - We apply 3.2 to the stable normal bundle of $M$ together with the duality results (mentioned in 2.3) implying $\delta_{-f}(Q, P)=\varepsilon \delta_{f}(P, Q)^{\nu}$.

Suppose now that $H_{*}(M ; \mathbf{Z})$ is torsion free and that for some $q<p<n / 2$ we have $H_{q}(M ; \mathbf{Z}) \approx H_{p}(M ; \mathbf{Z}) \approx \mathbf{Z}, H_{*}(M ; \mathbf{Z})=0$ for $q<*<p, *=q-1, *=p+1$. Then, in a minimal cell decomposition of $M$, there are two pairs of dual cells $e^{p}, e^{n-p}$ and $e^{q}$ and $e^{n-q}$ representing Poincaré dual generators in $H_{p}(M), H_{n-p}(M)$ and, respectively, $H_{q}(M)$, $H_{n-q}(M)$. As $e^{p}, e^{q}$ are attached in succession there is a relative attaching map $\delta: S^{p-1} \longrightarrow S^{q}$ and similarly $e^{n-q}$ and $e^{n-p}$ being attached successively we have another relative attaching map $\delta^{\prime}: S^{n-q-1} \longrightarrow S^{n-p}$.

In all this section $\varepsilon \in\{-1,+1\}$. Its presence in the formula below reflects an indeterminancy caused by the fact that if $\delta$ is the relative attaching map above, then there is a different minimal cell decomposition having as corresponding relative attaching map $-\delta$. 
COROLlaRY 4.2. - In the setting above $\delta$ and $\delta^{\prime}$ are (up to sign) independent of the minimal $C W$-decomposition used in their definition and $\{\delta\}=\varepsilon\left\{\delta^{\prime}\right\} \bmod \left(\operatorname{Im}\left(J^{q}\right)\right)$.

Proof. - The first part is immediate. We apply the corollary above to a perfect Morse function and its consecutive critical points $P, Q$ of indexes $p$ and $q$. Because of the first part we have $\delta_{f}(P, Q)=\delta$ and $\delta_{-f}(Q, P)=\delta^{\prime}$.

Remark 7. - (a) Corollary 4.2 can also be proven by purely homotopical methods. Surprisingly, it appears not to have been known before.

(b) For spaces more general than those appearing in 4.2 the relative attaching maps of the type of $\delta$ and $\delta^{\prime}$ depend on the specific minimal cell-decomposition used. Here is a relevant example (well known in the study of the Mislin genus [26]). Let $X=S^{9} \cup_{5 \nu} e^{13}$ and $X^{\prime}=S^{9} \cup_{13 \nu} e^{13}$ here $\nu \in \pi_{12}\left(S^{9}\right) \approx \pi_{3}^{S} \approx \mathbf{Z} / 24$ is a generator. These two complexes are not homotopy equivalent (even if they are so when localized at each prime $p$ ). However, it is easy to show that $X^{\prime} \vee S^{9} \simeq X \vee S^{9}$. Hence, we have two very different minimal cell decompositions for the space $Y=X \vee S^{9}$. In particular, if $N$ is a manifold with simply connected boundary having the homotopy type of $Y$ and of dimension greater than 26, each of these two cell decompositions is induced by a perfect Morse-Smale function. These two Morse-Smale functions are in different connected components of the space of perfect Morse-Smale functions of $N$. However, they are in the same component of the space of perfect Morse functions. Indeed, by a result of Matsumoto [25] the space of perfect Morse functions on a simply connected manifold with simply connected boundary, of dimension greater than 5 and with torsion free homology is connected (see also [2], for more general results [32]).

It is immediate to see that if $\Delta(P, Q) \notin \operatorname{Im}\left(J^{t}\right)$, then $k(P, Q)>t$. The obvious question that we consider now is what values can take the twisting $\Delta(P, Q)$.

Remark 8. - Certainly, there are examples when $\Sigma H(P, Q)$ is not vanishing, but $\Delta(P, Q)$ is null. An instructive example is that of $\mathbf{C P}^{n}$ together with a perfect Morse-Smale function. If $P$ and $Q$ are two arbitrary consecutive critical points of $f$, then $p=\operatorname{ind}(P)=\operatorname{ind}(Q)+2$ and $H(P, Q) \in \pi_{p-1}\left(\Sigma^{p-2} \Omega \mathbf{C P}^{n}\right) \approx \mathbf{Z}$ is a generator. On the other hand the difference $\delta_{f}(P, Q)-\delta_{-f}(Q, P)$ is nonzero iff $n$ is even (because in that case $w_{2} \neq 0$ ).

In the constructions below the strategy will be the following:

- Construct a certain Poincaré duality $C W$-complex $X$ with an explicit cell decomposition.

- Assuming $X$ smoothable, consider a perfect Morse-Smale function $f$ on $X$ which induces the fixed cell-decomposition at least below the middle dimension.

- Evaluate the restriction of the stable normal bundle of $X$ to the middle-dimensional skeleton.

- Identify two consecutive critical points $P$ and $Q$ and evaluate using $4.1,4.2$ or 3.2 the twisting $\Delta(P, Q)$.

\subsubsection{Some examples of Larry Smith}

Let $X=\left(S^{p} \vee S^{n-p}\right) \cup_{h} e^{n}$ where $h=\left[i_{1}, i_{2}\right]+i_{2} \circ x$ with $p<n / 2, i_{1}: S^{p} \longrightarrow S^{p} \vee S^{n-p}$ and $i_{2}: S^{n-p} \longrightarrow S^{p} \vee S^{n-p}$ the inclusions, [-,-] the Whitehead product, $x \in \pi_{n-1}\left(S^{n-p}\right)$ such that $x \notin \operatorname{Im}(J)$. Then, obviously $X$ is a Poincaré duality space, but it is not smoothable by 4.2 as the stable difference of the two relative attaching maps associated the first to the $p$ and 0 cells, and the second to the $n$ and $n-p$ cells, is equal to $x$.

Smith has proved this result by purely homotopical methods [34]. We indicate below a different, very short, purely homotopical proof of this same fact. It pinpoints the homotopical content of our Morse theoretical techniques. 
Assume that $X$ is smoothable. Thus, its stable normal bundle $\nu$ is orthogonal. Fix $m=\operatorname{rank}(\nu)$ and let $T^{\nu}(X)$ be the associated Thom space. It is Spanier-Whitehead dual to $X_{+}$. In particular, the first nontrivial relative attaching map in $T^{\nu}(X), \delta: S^{m+p-1} \longrightarrow S^{m}$ equals stably (up to sign) the top relative attaching map of $X, \delta^{\prime}: S^{n-1} \longrightarrow S^{n-p}$. Of course, $\delta^{\prime}=x$ hence $\delta=\varepsilon x$. Now, $\delta$ is already present in the Thom space, $T^{\nu}\left(S^{p}\right)$, of the restriction of $\nu$ to the bottom sphere $S^{p}$. By classical results (appearing already in [27] or [1]) we have that $T^{\nu}\left(S^{p}\right) \simeq S^{m} \cup_{\tau} e^{m+p}$ and $\tau \in \operatorname{Im}(J)$. As $\tau=\delta$ we are led to a contradiction.

Remark 9. - (a) Of course, if $x \in \operatorname{Im}\left(J_{P L}\right)$, then $X$ has the homotopy type of a $P L$ manifold [34].

(b) Clearly, one may extend the examples above by producing $(k-1)$-connected Poincaré duality complexes of dimension $n, 2 \leqslant k<n / 2$ with a relative attaching map of the top cell with respect to some $n-k$ cell that does not belong to $\operatorname{Im}(J)$. Of course, this is the content of Spivak's "first smoothing obstruction" [35]( see also [17]) which is thus recovered from 4.1. Notice, on the other hand, that Kervaire's original non-smoothable PL-manifold [16], as well as the examples of Eells and Kuiper [10], are not detected by these means. Indeed, the non-smoothable Poincaré spaces obtained by our methods are all stable in the sense that they remain non-smoothable after crossing with a sphere.

\subsubsection{Non-smoothable Poincaré spaces with vanishing Spivak first smoothing obstruction}

We construct here an example of a non-smoothable Poincaré complex whose non-smoothability can not be detected by the relative attaching maps of the top cell. These examples appear not to have been known before.

Take $q>2$ and $Z=\left(S^{2} \vee\left(S^{q} \cup_{\eta} e^{q+2}\right)\right) \cup_{[i, j]} e^{q+4}$ with $i: S^{2} \longrightarrow S^{2} \vee\left(S^{q} \cup_{\eta} e^{q+2}\right)$ the inclusion, $\eta \in \pi_{q+1}\left(S^{q}\right) \approx \mathbf{Z} / 2$ a generator and $j \in \pi_{q+2}\left(S^{q} \cup_{\eta} e^{q+2}\right)$ such that the image of $j$ via the pinching map $p: S^{q} \cup_{\eta} e^{q+2} \longrightarrow S^{q+2}$ is equal to twice a generator of $\pi_{q+2}\left(S^{q+2}\right)$. Consider $y \in \pi_{q+1}^{S}$ such that $2 y \notin \operatorname{Im}\left(J^{2}\right)$. Let $B F$ be the classifying space of stable spherical fibrations. As $\pi_{k}(B F)=\pi_{k-1}^{S}$ there is a spherical fibration given by the composition

$$
\mu: Z \stackrel{t}{\longrightarrow} S^{q+2} \stackrel{u}{\longrightarrow} B F_{m} \stackrel{v}{\hookrightarrow} B F \quad \text { with } y \simeq(v \circ u)^{*}
$$

$B F_{m}$ is the classifying space of spherical fibrations of fibre $S^{m-1}$ ( $m$ big enough) and $t$ is induced by the collapsing $S^{2} \vee\left(S^{q} \cup_{\eta} e^{q+2}\right) \longrightarrow S^{q+2}$. Let $N$ be a trivial, smooth thickening of $Z$ - this is a smooth manifold with boundary of dimension $n>2(q+4)+1$ having the homotopy type of $Z$ and which embedds in $S^{n}$ (by [39] it is unique up to isotopy). Let $N^{\prime}$ be the double of $N$. As $N^{\prime}$ is the union of two copies of $N$ pasted over $\partial N$, two copies of the spherical fibration $\mu$ can be also pasted together to give a new spherical fibration $\mu^{\prime}: N^{\prime} \longrightarrow B F_{m}$. The total space $K$ of $\mu^{\prime}$ is our example.

Remark 10. - There is another more elegant way to produce $\mu^{\prime}$ out of $\mu$. It was indicated to me by Mark Mahowald.

First a general remark of some independent interest (it appears to be folklore, for extensions see [19]). Because $N^{\prime}$ is the double of a trivial thickening, the suspension $\Sigma N^{\prime}$ splits as $\Sigma N \vee \Sigma^{2} N^{\#} \vee S^{n+1}$ with $N^{\#}$ homotopy equivalent to the complement of $N$ in $S^{n}$. Indeed, $N$ is embeddable in $S^{n}$, therefore we have $S^{n}=N \cup_{\partial N} N^{\#}$. The inclusions of $N$ and $N^{\#}$ in $S^{n}$ being homotopically trivial, we obtain a cofibration sequence $S^{n} \longrightarrow \Sigma \partial N \longrightarrow \Sigma N \vee \Sigma N^{\#}$. This cofibration sequence splits and thus $\Sigma \partial N \simeq \Sigma N \vee \Sigma N^{\#} \vee S^{n}$. Now $N^{\prime}=N \cup_{\partial N} N$ hence $\Sigma N^{\prime}=\Sigma N \cup_{\Sigma \partial N} \Sigma N$. The description of $\Sigma \partial N$ implies the claimed splitting. 
Consider $\mu^{*}: \Sigma N \longrightarrow B^{2} F$ whose adjoint is $\mu$. It extends trivially to

$$
\left(\mu^{*}\right)^{\prime}: \Sigma N^{\prime}=\Sigma N \vee \Sigma^{2} N^{\#} \vee S^{n+1} \longrightarrow B^{2} F
$$

Its adjoint gives $\mu^{\prime}$.

LEMMA 4.3. - The space $K$ does not have the homotopy type of a smooth manifold.

Proof. - Assume $K$ smoothable. Its stable normal bundle $\nu: K \longrightarrow \mathbf{B S O}\left(m^{\prime}\right), m^{\prime}>m+n$, has the property that if $\nu^{\prime}: K \longrightarrow \mathbf{B S O}\left(m^{\prime}\right) \longrightarrow B F_{m^{\prime}}$ is the associated spherical fibration, then $\left.\nu^{\prime}\right|_{N}+\mu=0$ in $[N, B F]$ (because this sum gives the Spivak spherical fibration of $N$ which is trivial). Therefore $\left.\nu^{\prime}\right|_{N}=-\mu$. Let $f: K \longrightarrow \mathbf{R}$ be a perfect Morse-Smale function inducing the standard cell decomposition given for $Z$ on the $(q+4)$-dimensional skeleton of $K, K^{(q+4)} \simeq Z$. We may assume also that $f$ is such that the critical points $P$ and $Q$ of indexes, repectively, $q+4$ and 2 are consecutive. Of course, $\delta_{f}(P, Q)=0$. We intend to evaluate $\Delta(P, Q)$. Consider the following diagram.

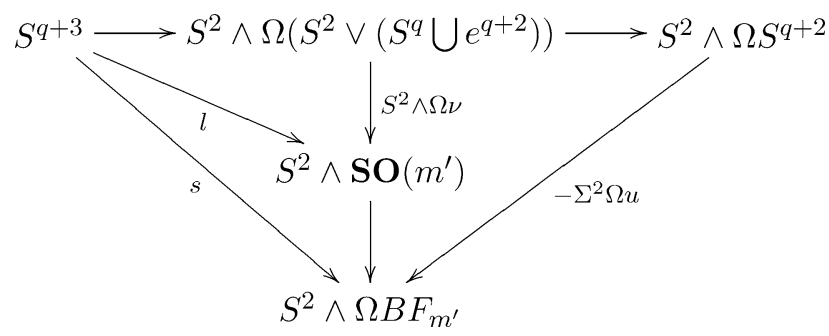

By the basic properties of the Whitehead product, the top horizontal composition is twice the inclusion of the bottom cell. This implies that $s=-2 \Sigma^{2} y$. Using 4.1 and the diagram below we see that $\delta_{-f}(Q, P)$ agrees stably (up to sign) with the composition $k \circ \Sigma^{m^{\prime}} l=k^{\prime} \circ \Sigma^{m^{\prime}} s$.

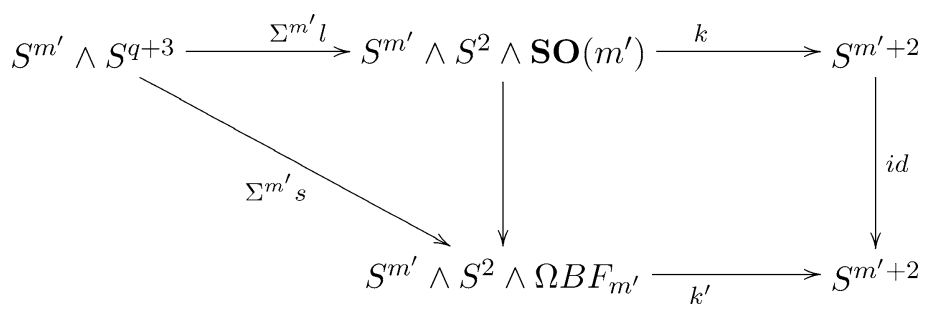

Here $k$ and $k^{\prime}$ are the double suspensions of the maps induced by the Hopf construction applied respectively to the actions $S^{m^{\prime}-1} \times \mathbf{S O}\left(m^{\prime}\right) \longrightarrow S^{m^{\prime}-1}$ and $S^{m^{\prime}-1} \times \Omega B F_{m^{\prime}} \longrightarrow S^{m^{\prime}-1}$. As $s$ is itself a double suspension we obtain that $k^{\prime} \circ \Sigma^{m^{\prime}} s$ is stably equal to the class of $s$ in $\pi_{q+1}(\Omega B F)=\pi_{q+1}^{S}$. Therefore, $\Delta(P, Q)=-2 y$ which leads to a contradiction.

Clearly the relative attaching maps of the top cell

$$
S^{n+m-1} \longrightarrow S^{n+m-2} \text { and } S^{n+m-1} \longrightarrow S^{n+m-q}
$$

vanish.

Remark 11. - (a) It is easy to see that if $y$ lifts to an element in $\pi_{*}(\Omega B P L)$, then $\mu$ and $\mu^{\prime}$ are PL-bundles and therefore $K$ is a PL-manifold. 
(b) Mark Mahowald has described another interesting non-smoothable Poincaré space that we now present (see also [24] p. 408).

Let $U$ be the total space of the spherical fibration classified by $\eta_{4}^{*} \in \pi_{17}\left(B F_{14}\right)$ where $\eta_{4}^{*}$ is the adjoint of the fourth element of Mahowald's family [23]. Recall that $\eta_{4} \notin \operatorname{Im}(J)$.

A cell decomposition of this space is as follows. Let $i_{n}$ be a generator of $\pi_{n}\left(S^{n}\right)$ and $\nu_{n}$ a generator of $\pi_{n+3}\left(S^{n}\right)$. Let $T=S^{13} \cup_{\nu_{13}} e^{17}$. The Whitehead product $\left[\nu_{13}, i_{13}\right]$ being null [38, 22] the relative Whitehead product $\left[i_{17}, i_{13}\right] \in \pi_{29}\left(T, S^{13}\right)$ pulls back to an element $z \in \pi_{29}(T)$ and $U=T \cup_{z} e^{30}$.

Notice that $\Sigma^{n} U$ splits as $S^{13+n} \cup_{\nu_{13+n}} e^{17+n} \cup_{i_{13+n} \circ \eta_{4}} e^{n+30}$.

The space $U$ is not smoothable. This is detectable by a top cell argument as follows.

We have $T=\Sigma T^{\prime}$. Thus, we may interpret $T$ as the cofibre of a trivial attaching map $T^{\prime} \longrightarrow *$. Therefore, $U \times S^{n}$ has a cone decomposition that starts with $T \vee S^{n}$ and has two other stages given by cofibration sequences

$$
T^{\prime} * S^{n-1} \longrightarrow T \vee S^{n} \longrightarrow U^{\prime} \text { and } S^{29} * S^{n-1} \longrightarrow U^{\prime} \longrightarrow U \times S^{n}
$$

By a Spanier-Whitehead duality argument, one sees that if $U$ and hence $U \times S^{n}$ are smoothable, then the relative attaching map $S^{n+29} \longrightarrow \Sigma^{n} T$ should not contain $\eta_{4}$.

Obviously, this example does not fit our Morse theoretic setting. However, a critical point approach is possible by using degenerate critical points as in [7].

\subsubsection{Morse-Smale functions with twisting realizing elements of $\operatorname{Im}\left(J^{q}\right)$}

We consider here the question of what elements in $\operatorname{Im}\left(J^{q}\right)$ can be realized as differences, $\Delta(P, Q)$, of attaching maps like in Corollary 4.1 .

LEMMA 4.4. - For any $x \in \operatorname{Im}\left(J^{q}\right), q \geqslant 2$, there is a smooth manifold $M$ and a MorseSmale function $f: M \longrightarrow \mathbf{R}$ with consecutive critical points $P$ and $Q$ such that $Q$ has index $q$ and $\delta_{f}(P, Q)=0, \delta_{-f}(Q, P)=\varepsilon x$.

Proof. - As $x \in \operatorname{Im}\left(J^{q}\right)$ there is $x^{1}: S^{p-1} \longrightarrow \Sigma^{q} \mathbf{S O}(m)$ such that $x$ is given by $J^{q}\left(x^{*}\right)$ where $x^{*}$ is the $q$ th order adjoint of $x^{1}$ and $m$ is large enough. Denote by $B S^{\prime}$ the $(p-q)$-dimensional skeleton of $\mathbf{B S O}(m)$ and let $S^{\prime}=\Omega B S^{\prime}$. Of course, $x^{1}$ lifts to an element $x^{\prime} \in \pi_{p-1}\left(\Sigma^{q} S^{\prime}\right)$.

There is a fibration $\Omega S^{q} * S^{\prime} \stackrel{j}{\longrightarrow} S^{q} \vee B S^{\prime} \longrightarrow S^{q} \times B S^{\prime}$ and a natural inclusion $\Sigma^{q} S^{\prime}=S^{q-1} * S^{\prime} \stackrel{i}{\hookrightarrow} \Omega S^{q} * S^{\prime}$. Let $x^{\prime \prime}=j \circ i \circ x^{\prime}$. Denote by $L$ the cofibre of $x^{\prime \prime}$. Fix a smooth, trivial, $n$-dimensional thickening of $L$ denoted by $H$ and let $H^{\prime}$ be its (smooth) double. We need to define a certain fiber bundle over $H^{\prime}$. Denote by $\mu: H \simeq L \longrightarrow$ BSO the bundle defined by the trivial extension of the map

$$
S^{q} \vee B S^{\prime} \hookrightarrow S^{q} \vee \mathbf{B S O}(m) \stackrel{c}{\longrightarrow} \mathbf{B S O}(m) \hookrightarrow \mathbf{B S O}
$$

( $c$ being the obvious collapsing). As discussed above one can extend $\mu$ to a bundle $\mu^{\prime}: H^{\prime} \longrightarrow \mathbf{B S O}$ and it lifts to a bundle $\tau: H^{\prime} \longrightarrow \mathbf{B S O}\left(m^{\prime}\right)$ with $m^{\prime}>n, m$. Let $G$ be the total space of the associated sphere bundle. As $H^{\prime}$ is smooth we see, by a transversality argument, that we may assume $\tau$ smooth and therefore $G$ is also smooth. There is a perfect MorseSmale function $f: G \longrightarrow \mathbf{R}$ such that the critical point $P$ of index $p$ and the critical point $Q$ of index $q$ corresponding to $S^{q} \hookrightarrow L \hookrightarrow H^{\prime}$ are consecutive, and such that the attaching map of the $p$-dimensional cell is given by $x^{\prime \prime}$. We have $\delta_{f}(P, Q)=0$. We now intend to compute $\delta_{-f}(Q, P)$. Let $\nu$ be the stable normal bundle of $G$. Obviously, its restriction to $L \subset H^{\prime}$ coincides with $-\mu$. Notice also that, as $\mu$ is null on $S^{q}, \Delta(P, Q)$ can be estimated by using the 
composition $S^{p-1} \stackrel{x^{\prime}}{\longrightarrow} S^{q} \wedge S^{\prime} \hookrightarrow S^{q} \wedge \Omega G$ instead of $H(P, Q)$. Indeed, the image of this composition by the map $S^{q} \wedge \Omega G \longrightarrow S^{q} \wedge \mathbf{S O}\left(m^{\prime}\right)$ coincides with the image of $H(P, Q)$. The composition $S^{q} \wedge S^{\prime} \hookrightarrow S^{q} \wedge \Omega G \stackrel{\Sigma^{q} \nu}{\longrightarrow} S^{q} \wedge \mathbf{S O}\left(m^{\prime}\right)$ is homotopic to the negative of the inclusion $S^{q} \wedge S^{\prime} \hookrightarrow S^{q} \wedge \mathbf{S O}\left(m^{\prime}\right)$.

Therefore, by applying 3.2, we see that $\delta_{-f}(Q, P)$ is (up to sign) equal to $J^{q}\left(x^{*}\right)=x$.

Remark 12. - It is likely that the Corollaries 4.1 and 4.2 as well as Proposition 3.2 have analogues in the $P L$ and Top categories.

\subsection{An extension of the Morse complex and detection of connecting flow lines}

The results of the second section can be used for the detection of critical points and connecting flow lines. Indeed, assume that $P, Q$ are consecutive critical points of the Morse-Smale function $f: M \longrightarrow \mathbf{R}$. If the suspension of the Hopf invariant $H(P, Q)$ is not zero, then there are flow lines connecting $P$ to $Q$. Of course, such connecting flow lines are already known to exist if $\delta_{f}(P, Q) \neq 0$ but, as seen before, this relative attaching map can vanish without $\Sigma H(P, Q)$ being also null.

\subsubsection{The energy functional on $\Omega S^{3}$}

This example has been suggested to me by Raoul Bott. Consider the energy functional $E$ on $\Omega\left(S^{3} ; u, v\right)$, the space of piecewise smooth curves connecting the points $u, v \in S^{3}$ (with $u$ and $v$ different and not antipodals). It is well known that $E$ is a perfect Morse function [28] whose critical points are the geodesics connecting $u$ and $v$. Fix two such geodesics $P$ and $Q$. Approximate $\Omega\left(S^{3} ; u, v\right)$ by a finite-dimensional manifold that contains $P$ and $Q$, on which the restriction of $E, E^{\prime}$, is still Morse, has critical points of the same index and the sets $\Omega^{a}=\left(E^{\prime}\right)^{-1}(-\infty, a]$ are compact for $a \leqslant b=\max \{E(P), E(Q)\}+\tau(\tau>0$ small $)$ and have the same homotopy type as $E^{-1}(-\infty, a]$ [28]. Fix a metric on $\Omega^{b}$ such that $E^{\prime}$ is Morse-Smale. We want to remark that $P$ and $Q$ are connected by some flow lines of the flow induced by $-\nabla E^{\prime}$. Notice that $\Omega\left(S^{3} ; u, v\right)$ is homotopy equivalent to $\Omega S^{3}$. Assume $R$ is another critical point of $E$ in $\Omega^{b}$ such that $\operatorname{ind}(P)=\operatorname{ind}(R)+2$, then $\delta_{f}(P, R)=0$. However, $\Sigma H(P, R) \neq 0$. This implies, by transitivity, that $P$ and $Q$ are also connected by some flow line.

\subsubsection{Extension of the Morse complex}

The "detection" arguments above can be pursued further. For example, if $M$ is simplyconnected and $P, Q$ and $Q, R$ are two pairs of consecutive critical points for which the homology classes $[h(P, Q)],[h(Q, R)] \in H_{*}(\Omega M ; \mathbf{Z} / 2)$ are known and if $[h(P, Q)] \bullet[h(Q, R)] \neq 0$ it follows from 3.3 that there is at least another critical point $Q^{\prime}$ that is connected via possibly broken flow lines to both $P$ and $R$ (• being here the Pontryagin product).

Here is a way to encode in a somewhat global fashion the type of information given by 3.3.

As before $f: M \longrightarrow \mathbf{R}$ is a Morse-Smale function, constant, regular and maximal on $\partial M$. Let $a_{1}<a_{2}<\cdots<a_{r}$ be a set $A$ of real numbers such that the critical values of $f$ all appear among the $a_{i}$ 's as well as $f(\partial M)$. Let $\mathcal{C}_{k}=\Omega_{*}^{f r}(\Omega M)<X \in f^{-1}\left(a_{k}\right): \nabla f(X)=0>$ (where $R<X_{1}, \ldots, X_{n}>$ is the free $R$-module generated by $\left.X_{1}, \ldots, X_{n}\right)$. If $X$ is a critical point let $x$ be its index. Let $d: \mathcal{C}_{i} \longrightarrow \mathcal{C}_{i-1}$ be the unique $\Omega_{*}^{f r}(\Omega M)$-module morphism given by

$$
d(P)=\sum_{X \in f^{-1}\left(a_{i-1}\right), \nabla f(X)=0}(-1)^{p x}[Z(P, X)] X .
$$

COROLLARY 4.5. - The graded $\Omega_{*}^{f r}(\Omega M)$-module $\left(\mathcal{C}_{*}, d\right)$ is a chain complex. 
Proof. - Notice that any pair of critical points $y \in f^{-1}\left(a_{i}\right), x \in f^{-1}\left(a_{i-1}\right)$ are consecutive and apply 3.3.

Remark 13. - (a) A somewhat easier to handle complex is obtained by replacing $\Omega_{*}^{f r}(\Omega M)$ with the Pontryagin ring $H_{*}(\Omega M ; \mathbf{Z} / 2)$ and using instead of $d$,

$$
d^{\prime}(P)=\sum_{X \in f^{-1}\left(a_{i-1}\right), \nabla f(X)=0} H^{\prime}(P, X) X
$$

where, for two consecutive critical points $P$ and $Q$, we denote by $H^{\prime}(P, Q)$ the number of elements in $Z(P, Q)$ if $p=q+1$ and the homology class of $H(P, Q)$ in $H_{p-q-1}(\Omega M ; \mathbf{Z} / 2)$ if $p>q+1$. If $f$ is a self indexed function and $A=\mathbf{N}$, then this complex is the Morse complex of $f$ tensored with $H_{*}(\Omega M ; \mathbf{Z} / 2)$ (recall that $M$ is simply connected).

(b) It would be interesting to know whether one can deduce the existence of the discussed chain complex by analytic methods.

\subsubsection{Fusion of critical points}

One initial motivation for this work was the problem of constructing functions with the least possible number of (possibly degenerate) critical points on a given smooth manifold $M$. It is well-known that a strict lower bound for this number is the Lusternik-Schnirelmann category $\operatorname{cat}(M)$ of $M$ [21]. It was shown in [8] that when $M$ is 2 -connected, and $k \geqslant \operatorname{dim}(M)$ there is a function on $M \times D^{k}$ regular, maximal and constant on $\partial\left(M \times D^{k}\right)$ which realizes the lower bound given by the category up to one unit. The next step is to understand when two consecutive critical points of a given function can be "fused" together. More precisely, let $f: M \longrightarrow \mathbf{R}$ be a smooth function and let $P, Q$ be consecutive critical points of $f$. The question is whether there is a function $f^{\prime}$ equal to $f$ in the exterior of some neighborhood $U$ of the closure of the points situated on flow lines connecting $P$ to $Q$ and having just one critical point in $U$. It is natural to first assume that $P$ and $Q$ are consecutive, non-degenerate critical points of indexes, respectively, $p$ and $q$, and that, with respect to some fixed metric on $M, f$ is Morse-Smale. One can also weaken the question by asking $P$ and $Q$ to be fused to a "reasonable" critical point [8], a class that contains all critical points of locally analytic functions.

LEMma 4.6. - Assume that $f^{-1}(-\infty, f(Q))$ has the homotopy type of a $k$-dimensional $C W$-complex with $k<q-1$. If $H(P, Q) \neq 0$, then $P$ and $Q$ cannot be fused to a reasonable critical point.

Proof. - This is a simple consequence of some results in [8]. First, as $P$ and $Q$ are consecutive and $f$ is Morse-Smale we may assume that $f(P)>f(Q)$ and that $P$ and $Q$ are the only critical points in $f^{-1}[f(Q), f(P)]$. If $P$ and $Q$ can be fused to a reasonable critical point, one deduces [8] a cofibration sequence $Z \longrightarrow M^{\prime} \longrightarrow M^{\prime \prime \prime}$ where $M^{\prime}=f^{-1}(-\infty, f(Q)-\tau]$, $M^{\prime \prime \prime}=f^{-1}(-\infty, f(P)+\tau]$ with $\tau>0$ small. Let $M^{\prime \prime}=f^{-1}(-\infty, f(Q)+\tau]$. The composition $S^{p-1} \longrightarrow M^{\prime \prime} \stackrel{\nabla}{\longrightarrow} S^{q} \vee M^{\prime \prime}$ is homotopic to $S^{p-1} \stackrel{\nabla^{\prime}}{\longrightarrow} S^{q} \vee S^{p-1} \stackrel{i d \vee \alpha(P)}{\longrightarrow} S^{q} \vee M^{\prime \prime}$ where $\nabla^{\prime}$ is the coaction associated to the cofibration sequence $S^{q-1} \longrightarrow Z \longrightarrow S^{p-1}$. As

$$
\pi_{p-1}\left(S^{q} \vee S^{p-1}\right)=\pi_{p-1}\left(S^{q}\right) \oplus \pi_{p-1}\left(S^{p-1}\right)
$$

it follows that $H(P, Q)$ vanishes.

Remark 14. - Controlling the behavior of Hopf invariants has recently become a key tool in the homotopical study of the Lusternik-Schnirelmann category. Results based on this technique are the negative solution of the Ganea conjecture by Iwase [14], the examples of Roitberg 
showing that the L.S.-category is not generic in the sense of the Mislin genus [30] and the examples of Stanley of spaces of category $n$ but cone-length $n+1$ [36]. In all these examples the non-vanishing of certain Hopf invariants associated to the attachment of a cell is used to deduce that the L.S.-category is increased by the attachment of that cell. This is clearly also the homotopical content of the lemma above.

\section{Acknowledgements}

I thank Raoul Bott, Fred Cohen, Pascal Lambrechts, Chuck McGibbon and Alberto Verjovsky for useful discussions as well as John Harper whom I also thank for pointing out [34]. I am most grateful to Mark Mahowald for his valuable suggestions and for his encouragement. I also thank the referee for some useful comments and, in particular, for drawing my attention to the need to choose a "correct" base-point at the beginning of Section 3 .

\section{REFERENCES}

[1] Adams F., On the groups $J(X)$. IV, Topology 5 (1966) 21-71.

[2] Agoston M.K., On handle decompositions and diffeomorphisms, Trans. Amer. Math. Soc. 137 (1969) 21-26.

[3] Arkowitz M., The generalized Whitehead product, Pacific J. Math. 12 (1962) 7-23.

[4] Cohen R.L., Jones J.D.S., Segal G.B., Morse theory and classifying spaces, Preprint.

[5] Cohen R., Jones J.D.S., Segal G., Floer's Infinite Dimensional Morse Theory and Homotopy Theory, The Floer Memorial Volume, Birkhauser, 1995.

[6] Cornea O., Homotopical dynamics: suspension and duality, Ergodic Theory Dynamical Systems 20 (2000) 379-391.

[7] Cornea O., Spanier-Whitehead duality and critical points, in: Homotopy Theory via Algebraic Geometry and Group Representations, in: Contemp. Math., Vol. 220, American Mathematical Society, 1998, pp. 47-63.

[8] Cornea O., Cone-decompositions and degenerate critical points, Proc. London Math. Soc. 77 (1998) 437-461.

[9] Dula G., On conic spaces, in: Lecture Notes in Math., Vol. 1474, Springer, 1991, pp. 38-58.

[10] Eells J., KuIPER N.H., Manifolds which are like projective planes, Pub. IHES 14 (1962) 5-46.

[11] FranKS J., Morse-Smale flows and homotopy theory, Topology 18 (1979) 199-215.

[12] Ganea T., Cogroups and suspension, Invent. Math. 9 (1970) 185-197.

[13] Ganea T., A generalization of the homology and homotopy suspension, Comment. Math. Helv. 39 (1965) 295-322.

[14] IwASE N., Ganea's conjecture on the Lusternik-Schnirelmann category, Bull. London Math. Soc. 30 (1998) 623-634.

[15] Kahn D., Priddy S., Applications of the transfer to stable homotopy, Bull. Amer. Math. Soc. 78 (1972) 981-987.

[16] Kervaire M., A manifold which does not admit any differentiable structure, Comment. Math. Helv. 34 (1960) 257-270.

[17] Kervaire M., Milnor J., Groups of homotopy spheres. I, Ann. of Math. 77 (1963) 504-537.

[18] KLEIN J., Higher Reidemeister torsion and parametrized Morse theory, Rend. Circ. Mat. Palermo (2) Suppl. 30 (1993) 15-20.

[19] KLEIN J., Poincaré duality embeddings and fiberwise homotopy theory, Topology, to appear.

[20] KNAPP K., On the bi-stable J-homomorphism, in: Lecture Notes in Math., Vol. 763, Springer, 1979, pp. 13-22.

[21] Ljusternik L., Schnirelmann L., Methodes topologiques dans les problèmes variationels, Hermann, Paris, 1934.

[22] Mahowald M., Some Whitehead products in $S^{n}$, Topology 4 (1965) 17-26.

[23] Mahowald M., A new infinite family in ${ }_{2} \pi_{*}^{S}$, Topology 16 (1977) 249-256.

$4^{\mathrm{e}}$ SÉRIE - TOME $35-2002-\mathrm{N}^{\circ} 4$ 
[24] Mahowald M., Thompson R.D., The EHP sequence and periodic homotopy, in: James I.M. (Ed.), Hanbook of Algebraic Topology, Elsevier, Amsterdam, 1995, pp. 397-423.

[25] Matsumoto T., On the minimal ordered Morse functions, Publ. RIMS Kyoto Univ. 14 (1978) 673684.

[26] McGibbon CH., The Mislin genus of a space, in: CRM Proc. Lecture Notes, Vol. 6, American Mathematical Society, 1994, pp. 75-102.

[27] MiLnOR J., On the cobordism ring $\Omega^{*}$ and a complex analogue, Amer. J. Math. 82 (1960) 505-521.

[28] Milnor J., Morse Theory, in: Ann. of Math. Studies, Princeton University Press, 1963.

[29] Quillen D.G., The Adams conjecture, Topology 10 (1970) 67-80.

[30] RoITBERG J., Lusternik-Schnirelmann category of some infinite complexes, Topology 39 (2000) 95101.

[31] Schwarz M., Morse Homology, in: Progress in Mathematics, Vol. 111, Birkhauser, 1993.

[32] Sharko V., Functions on Manifolds, in: Transl. of Math. Monographs, Vol. 131, American Mathematical Society, 1993.

[33] Smale S., Differentiable dynamical systems, Bull. Amer. Math. Soc. 73 (1967) 747-817.

[34] Sмiтh L., Manifolds with few cells and the stable homotopy of spheres, Proc. Amer. Math. Soc. 31 (1) (1972) 279-285.

[35] SPIVAK M., Spaces satisfying Poincaré duality, Topology 6 (1967) 77-101.

[36] STANLEy D., Spaces of Lusternik-Schnirelmann category $n$ and of cone-length $n+1$, Topology 39 (2000) 985-1019.

[37] Sullivan D., Genetics of homotopy theory and the Adams conjecture, Ann. of Math. 100 (1974) $1-79$.

[38] Toda H., Composition Methods in the Homotopy Groups of Spheres, in: Ann. of Math. Studies, Vol. 49, Princeton Univ. Press, 1962.

[39] Wall C.T.C., Classification problems in differential topology IV, Topology 3 (1966) 73-94.

[40] Whitehead G., Elements of Homotopy Theory, in: Grad. Texts in Math., Vol. 61, Springer, 1978.

[41] Witten E., Supersymmetry and Morse theory, J. Differential Geom. 17 (1982) 661-692. 